\title{
Loss of rice PARAQUAT TOLERANCE 3 confers enhanced resistance to abiotic stresses and increases grain yield in field
}

\author{
Alamin Alfatih, Jiu Wu, Sami Ullah Jan, Zi-Sheng Zhang, Jing-Qiu Xia, and Cheng-Bin \\ Xiang
}

School of Life Sciences and Division of Molecular \& Cell Biophysics, Hefei National Science Center for Physical Sciences at the Microscale, University of Science and Technology of China, The Innovation Academy of Seed Design, Chinese Academy of Sciences, Hefei, Anhui Province 230027, China.

Correspondence to Cheng-Bin Xiang (email: xiangcb@ustc.edu.cn)

\begin{abstract}
Plants frequently suffer from environmental stresses in nature and have evolved sophisticated and efficient mechanisms to cope with the stresses. To balance between growth and stress response, plants are equipped with efficient means to switch off the activated stress responses when stresses diminish. We previously revealed such an off-switch mechanism conferred by Arabidopsis PQT3, knockout of which significantly enhances resistance to abiotic stresses. To explore whether the rice homolog OSPQT3 is functionally conserved, we generated three knockout mutants with CRISPR-Cas9 technology. The OsPQT3 knockout mutants (ospqt3) display enhanced resistance to oxidative and salt stress with elevated expression of $O s G P X 1, O s A P X 1$, and $O s S O D 1$. More importantly, the ospqt3 mutants show significantly enhanced agronomic performance with higher yield compared with the wild type under salt stress in greenhouse as well as in field conditions. We further showed that $O S P Q T 3$ was rapidly down regulated in response to oxidative and other abiotic stresses as AtPQT3. Taken together, these results support our previous findings that AtPQT3 acts as an off-switch in stress response, which is well conserved in rice. Therefore, PQT3 locus provides a promising candidate for crop improvement with enhanced stress resistance by gene editing technology.
\end{abstract}

Keywords: paraquat, oxidative stress, salt stress, OsPQT3, E3 ubiquitin ligase, reactive oxygen species (ROS), rice 
bioRxiv preprint doi: https://doi.org/10.1101/2020.02.22.961151; this version posted February 23, 2020. The copyright holder for this preprint (which was not certified by peer review) is the author/funder. All rights reserved. No reuse allowed without permission. 


\section{INTRODUCTION}

Oxidative stress is a common component to all environmental stresses that disturb cellular oxygen metabolism (Das and Roychoudhury, 2014). Plants have evolved wellcoordinated defense mechanisms to cope with oxidative stress generated under wide-range abiotic stresses (Mittler et al., 2004). Two eminent types of defensive responses are enzymatic response and non-enzymatic response. The first type of response comprises of cellular enzymes like superoxide dismutase (Li et al., 2011), peroxidase (POD), catalase (CAT), ascorbate peroxidase (APX), peroxiredoxin Q (PRXQ), and glutathione peroxidase (GPX) which protect cell by engaging toxic ions entering the cell or generated within the cell in stress conditions (Apel and Hirt, 2004; Mittler et al., 2004; Takahashi and Asada, 1988). Such antioxidant enzymes are helpful in achieving tolerance against oxidative stress (Ye and Gressel, 2000). The later type of defense response include non-enzymatic cellular components such as ascorbate and glutathione that, in majority, serves as antioxidants as well as signals to modulate cellular functions in response to abiotic or biotic stimuli (Moon et al., 2004; Vierstra, 2009).

Paraquat (N,N'-dimethyl-4,4'-bipyridinium dichloride, PQ), a widely used non-selective and rapid-acting herbicide, is a highly effective inducer of oxidative stress in plants. Paraquat diverts electrons from photosystem I towards molecular oxygen, generating superoxide, which is rapidly converted by superoxide dismutase to hydrogen peroxide, and thus raising the concentration of reactive oxygen species (ROS) to toxic or even lethal level in green plants (Summers, 1980), which alters normal cellular homeostatic condition (Haley, 1979; Miller et al., 2010). The accumulated ROS damages macromolecules and biological membranes, induces cell senescence, and causes irreversible injuries to cell and cellular components (Gill and Tuteja, 2010). It is well established that ROS concentration significantly and rapidly increases under numerous stresses such as drought, salt and paraquat-induced stress (Leshem et al., 2006; Miller et al., 2010; Van Breusegem et al., 2008).

Paraquat is also a commonly used oxidative stress inducer in the laboratory for mutant screen (Chen et al., 2009; Kurepa et al., 1998). We previously isolated paraquat resistance mutants (Xi et al., 2012) and reported AtPQT3 encoding an E3 ubiquitin ligase, which turns off or negatively regulates the response activated against oxidative stress (Luo et al., 2016). AtPQT3 interacts with PRMT4b that help plants tolerate oxidative stress by methylating histones on the chromatin of $A t G P X 1$ and AtAPXI, thus enhancing their expression and 
leading to stress resistance (Luo et al., 2016). In the process of ubiquitination, E3 ubiquitin ligases regulate downstream signaling processes in biotic and abiotic stress response through mediating transcription factors (TFs) or vesicle trafficking (Zhou et al., 2017). An example reported earlier showed that E3 ubiquitin ligase RING-type MIEL1 interacts with AtMYB30 leading to proteasomal degradation of AtMYB30, thus causing Arabidopsis defense attenuation (Marino et al., 2013). Another RING-type E3 ubiquitin ligase EIRP1 interacts with WRKY11 in Vitis pseudoreticulata promoting defense against fungal infection (Yu et al., 2013). Similarly, $O s H C I 1$ is responsive to temperature variation in rice. When overexpressed, OsHCIl showed improved tolerance to heat in Arabidopsis (Lim et al., 2013). Lately, it was reported that the E3 ubiquitin ligase SOR1 modulates ethylene response by regulating IAA/Aux protein stability in rice roots (Chen et al., 2018).

In this study, we investigate whether OSPQT3, the rice homolog of AtPQT3, is functionally conserved or not. Our results show that $O S P Q T 3$ negatively regulates oxidative stress response. Loss-of-OsPQT3 confers salt and paraquat tolerance as demonstrated by the ospqt3 knockout mutants. The expression of OsAPX1, OsGPX1 and OsSOD1 was increased in ospqt3 as compared to that in the wild type. Our findings suggest that OsPQT3 shares functional similarity with $A t P Q T 3$ as both are negative regulators of oxidative stress response. Moreover, ospqt3 mutants exhibited significantly enhanced growth and grain yield of rice under both normal and stressed conditions, therefore providing a candidate gene for improving abiotic stress tolerance and yield of crops via gene editing.

\section{RESULTS}

\section{Loss-of-OsPQT3 confers enhanced tolerance to paraquat and salt}

Previous studies in dicots have demonstrated that the atpqt3 plays important roles in the response of Arabidopsis thaliana to oxidative stress (Luo et al., 2016). However, its roles in rice remain unknown. We resorted to CRISPR-Cas9 technology to generate mutations in OsPQT3 gene and obtained 3 independent mutants (ospqt3-1, ospqt3-2, and ospqt3-3). The first mutant (ospqt3-1) had a 5-base deletion in the first exon, the second mutant (ospqt3-2) gained one base in the first exon, and the third mutant (ospqt3-3) gained one base in the second exon. Consequently, the open reading frame of Os10g29560 was interrupted in all three mutants as confirmed by genomic DNA sequencing analyses (Figure 1). Therefore, we successfully generated three independent null mutants for further study. 
We first checked the germination rate in response to paraquat and salt stress. The seeds of ospqt3-1, ospqt3-2, ospqt3-3 and wild type were germinated in MS medium with or without $0.1 \mu \mathrm{M}$ paraquat for eight days. The germination of wild type and mutants revealed no difference in the absence of paraquat. However, in the presence of $0.1 \mu \mathrm{M}$ paraquat the germination of ospqt3 mutants were significantly higher than the wild type plants (Figure 2A). Similarly, the germination rate of wild type plants were severely decreased in the medium containing $175 \mathrm{mM} \mathrm{NaCl}$ compared to ospqt3 mutant (Figure 2A). This result suggests that $O S P Q T 3$ play a negative role in seed germination in response to abiotic stresses.

Next, we checked the survival ratio of seedlings in response to paraquat and salt stress. The seeds of ospqt3-1, ospqt3-2, ospqt3-3 and wild type were germinated in MS medium with or without $0.1 \mu \mathrm{M}$ paraquat for two weeks. All the wild type and mutants survived on MS medium without apparent differences. However in the presence of paraquat, the survival ratio ospqt 3 mutants was more than $80 \%$ in contrast to less than $20 \%$ of the wild type (Figure 2B). This result suggests that the mutants are more tolerant to the oxidative stress generated by paraquat in comparison to wild type. When germinated in presence of $175 \mathrm{mM} \mathrm{NaCl}$ for 2 weeks, the survival ratio of the ospqt3 mutants was around $80 \%$, while the wild type had only $20 \%$ survival rate (Figure 2B). This result indicates that OSPQT3 plays negative roles in salt stress tolerance in rice, which is consistent to the Arabidopsis pqt3 mutant (Luo et al., 2016). Furthermore, the growth of the survived wild type seedlings was significantly inhibited in the presence of paraquat and salt compared with the mutants (Figure 2C).

\section{The ospqt3 mutants show improved salt stress resistance in the seedling and vegetative stages.}

To evaluate the attributes of salt resistance in the ospqt3 mutant seedlings, we treated six-day-old seedlings of ospqt3-1, ospqt3-2, and ospqt3-2, and wild type (WT) with 0, 75, $100 \mathrm{mM} \mathrm{NaCl}$ in hydroponic solution for 10 days. Both ospqt3 mutants and WT seedlings grew well under $0 \mathrm{mM} \mathrm{NaCl}$ although the wild type shoot was a little taller than the mutant. After exposure to $75 \mathrm{mM}$ or $100 \mathrm{mM}$ salt stress, most ospqt3 mutants seedlings remained green and showed continuous growth, whereas WT seedlings at $75 \mathrm{mM} \mathrm{NaCl}$ were $20 \%$ dead while at $100 \mathrm{mM} \mathrm{NaCl}$ were almost $90 \%$ dead (Figure 3A and $\mathrm{B}$ ). The survival rate of the WT seedlings were $10 \%$, but the survival rate of ospqt3 mutant seedlings reached $80 \%$ under $100 \mathrm{mM} \mathrm{NaCl}$ treatment (Figure 3B). 
To evaluate salt tolerance of ospqt 3 mutants grown in soil, we grew plants in the greenhouse and irrigated 4-week-old plants of WT, ospqt3-1, ospqt3-2, and ospqt3-3 with 0 $\mathrm{mM}$ or $200 \mathrm{mM} \mathrm{NaCl}$ for 1 week (Figure 3C). The result shows that, the mutant plants were significantly more tolerant to the salt stress compared with the WT plants. The survival rate of the ospqt 3 mutants was more than $50 \%$ while $21 \%$ of WT survived under $200 \mathrm{mM} \mathrm{NaCl}$ treatment (Figure 3D). Collectively, these results suggest that ospqt3 mutants are more resistant to salt stress than the WT at the seedling and vegetative growth stages.

\section{The ospqt3 mutants exhibit improved grain yield in field conditions.}

To test the field performance of ospqt3 mutants, we conducted field trials of WT (ZH11), ospqt3-1, ospqt3-2, and ospqt3-3 mutant plants at two different locations in China: Hefei, Anhui Province from April to August in 2019 (Figure 4) and Hainan Island from November to April in 2019 (Figure 5). Figure 4A shows the typical plant of WT and ospqt3 mutants from the normal field trial in Hefei and Figure 4B shows the panicle size of WT and ospqt3 mutant plants. The ospqt3 mutants exhibited significantly increased grain yield by $11.4 \%$ (Figure 4C), tiller number by 25\% (Figure 4D), panicle length by $12.2 \%$ (Figure 4E), seed setting rate by $6.7 \%$ (Figure $4 \mathrm{~F}$ ), and primary branch number by $14.2 \%$ (Figure $4 \mathrm{G}$ ), except for plant height (Figure 4H). The field trial results in Hainan Island is shown in Figure 5. A typical panicle is shown for different genotypes in Figure 5A. Again, the ospqt3 mutants exhibited significantly increased grain yield by $15 \%$ (Figure 5B), tiller number by $20 \%$ (Figure 5C), and panicle length by $12.5 \%$ (Figure 5D) but no difference was found in plant height (Figure 5E). Taken together, these results suggest that ospqt3 mutants exhibit not only paraquat and salt tolerance, but also enhanced grain yield, making it a promising candidate for crop improvement.

\section{The ospqt3 mutants exhibit enhanced salt tolerance and significantly increase grain yield in greenhouse.}

To evaluate the grain yield under salt stress, we carefully examined the ospqt 3 mutants in the greenhouse. In the normal growth conditions the mutant plants had a little larger panicle than WT control (Figure 6A). However, in the presence of salt stress the mutant had significantly larger panicle compared to WT plants (Figure 6B). A much more significant difference was observed in the salt stress condition which, the grain yield of ospqt3 mutant increased by more than $30 \%$ of the wild type (Figure 6C). 
Tillering is one of the most vital agronomic traits related to grain production in rice ( $\mathrm{Li}$ et al., 2003). The effective tillers number of mutant plants grown in greenhouse in the absence of salt stress increased $14 \%$ over the WT control. While in the presence of $70 \mathrm{mM} \mathrm{NaCl}$, the ospqt3 mutant had significantly increased tillers number by $33.3 \%$ more than WT plants (Figure 6D). Consistent with these results, ospqt3 mutant grown in the field shown more tiller numbers than the wild type (Figure 4 and 5). Apparently, increased tiller number is one contributor to the increased yield. The increased panicle size of the ospqt3 mutant rice was largely contributed by the increased number of primary branches (Figure 6E and F). The seed setting rate under well irrigated conditions shows no obvious difference between the ospqt3 mutants and WT while in the presence of salt stress, the seeds setting rate of the ospqt3 mutants was increased by $50 \%$ over the WT plants (Figure 6G). Finally, ospqt3 mutants grown in normal conditions had no clear differences in the plant height with comparison to the WT plants. However, in the salt stress conditions the plant height of the WT plants were decreased by $9 \%$ compared to the ospqt 3 mutants (Figure $6 \mathrm{H}$ ).

\section{$O S P Q T 3$ is rapidly down-regulated by stress treatments.}

Quantitative PCR was used to check the expression level of OsPQT3 in the seedling stage, vegetative and premature stages. The $O s P Q T 3$ expression was significantly higher in the seedling shoot as compared to root. While in the vegetative and premature stages the expression was higher in root (Figure 7A). As predicted, the expression of OsPQT3 was rapidly down-regulated upon paraquat treatment (Figure 7B), which supports that OSPQT3 serves as negative regulator for oxidative tolerance in plants. Similarly, the expression of OsPQT3 was down-regulated by $\mathrm{H}_{2} \mathrm{O}_{2}$, mannitol, drought, $\mathrm{CdCl}_{2}$, and salt treatment (Figure 7B-G). Relatively, paraquat and drought rapidly and significantly reduced expression of OsPQT3 and kept the transcript level low throughout the treatments (Figure 7B and E). Although the OSPQT3 expression was rapidly down-regulated at 0.5 and 1.0 hours of the treatment with $\mathrm{CdCl}_{2}$, however, OsPQT3 transcript level was recovered at 3 and 6 hours of the treatment with $\mathrm{CdCl}_{2}$ (Figure 7E). These results are consistent with that of the Arabidopsis $P Q T 3$, indicating $P Q T 3$ may have conserved functions in rice and Arabidopsis plants.

\section{OsAPX1, OsGPX1, and OsSOD1 are up-regulated in ospqt3 mutants.}


Enzymatic defense systems are crucial for eliminating ROS. Transcript levels of glutathione peroxidase $(O s G P X)$, ascorbate peroxidase $(O s A P X)$, plastidic $\mathrm{Cu} / \mathrm{Zn} S O D$ (OsCSD2), Cu/Zn OsSOD (OsCSD1), catalase (OsCAT), cytosolic mitochondrial MnSOD $(O s M S D)$, glutaredoxin $\mathrm{C}(O s G R X C)$, peroxiredoxin $\mathrm{Q}(O s P R X Q)$ 2-Cys peroxiredoxin $\mathrm{B}$ $(O s 2 C P B)$, and atypical Cys-His rich thioredoxin (OsACHT) were analyzed through quantitative RT-PCR in ospqt3 mutants and WT. We found that the transcript levels of OsGPX1, OsAPX1 and OsSOD1 were significantly upregulated in ospqt3 mutants as compared to that of WT under normal conditions (Figure 8A). The up-regulated transcript levels of $O s G P X 1, O s A P X 1$ and $O s S O D 1$ indicate that they are responsible for the increased resistance against oxidative stress in the mutants.

We also compared the enzyme activity of APX, GPX and SOD between WT and ospqt3 mutants. The wild type had lower enzyme activity of APX, GPX and SOD compared with pqt3 mutants (Figure 8B). These results are consistent with that found in the Arabidopsis pqt3 mutant, which also supports that the $P Q T 3$ has a conserved function in rice and Arabidopsis plants. Taken together, these results demonstrate that $O S P Q T 3$ is a negative regulator in oxidative response as AtPQT3 in Arabidopsis thaliana through similar molecular mechanism.

\section{DISCUSSION}

In this study, we assessed the function of $O S P Q T 3$ in rice. Our results show that ospqt3 mutants exhibit not only enhanced resistance to oxidative and salt stress but also increased grain yield of rice. These findings highlight $O S P Q T 3$ as a promising candidate gene in crop improvement. The paraquat and salt tolerant phenotypes of ospqt3 mutants are contributed mainly by antioxidation enzymes.

The ospqt3 mutants showed a high seed germination rate and a high survival rate during seeding stage in the presence of paraquat and salt compared to WT plants (Figure 2). Moreover, the expression level of $O S P Q T 3$ was significantly decreased in response to multiple different stresses such as paraquat, $\mathrm{H}_{2} \mathrm{O}_{2}$, mannitol, drought, $\mathrm{CdCl}_{2}$ and $\mathrm{NaCl}$, consistent with the role of $O S P Q T 3$ as a negative regulator. Finally, increased transcription level of $O s A P X, O s G P X$ and $O s S O D$ and increased activities of OsAPX, OsGPX and OsSOD enzymes observed in the ospqt3 mutant plants (Figure 8) are also in agreement with their enhanced tolerance against oxidative stress. Higher concentration of ROS poses direct impact upon biological membranes, disrupts macromolecules, promotes cell senescence, induces 
irreversible cellular damages and eventually leads to cell death (Gill and Tuteja, 2010). Several other ROS-scavenging enzymes, in addition to $A P X$, including $G P X, C A T, \operatorname{Pr} x R$, and GST are involved in scavenging excessive $\mathrm{H}_{2} \mathrm{O}_{2}$ generated under wide range of abiotic stresses (Mittler et al., 2004). These enzymes play their part in breaking $\mathrm{H}_{2} \mathrm{O}_{2}$ down to water and thus reduce $\mathrm{H}_{2} \mathrm{O}_{2}$ concentration (Dixon and Edwards, 2010; Mittler et al., 2004). It is axiomatic that up-regulating such ROS scavenger-encoding genes will speed up the process of reducing toxic ROS concentration. In ospqt3 mutants multiple antioxidation enzymes are maintained at a higher level, which enhances the ability to resist abiotic stress. Biotic and abiotic stresses restrict crop productivity. Drought and salt are the most influential abiotic stresses that severely restrict crop productivity (Boyer, 1982; Jamil et al., 2011; Rockström and Falkenmark, 2000). The ospqt3 mutants exhibited enhanced salt tolerance at all growth stages tested (germination, vegetative and reproductive) (Figure 2, Figure 3 and Figure 6). We observed that loss-of-OSPQT3 affected grain yield components like effective tillers number, panicle length, primary branch number, seeds setting rate, and ultimately grain yield per plant (Figure 4 and Figure 5).

Based on our findings, ospqt3-enhanced grain yield in rice is primarily achieved through the following major factors. Firstly, ospqt3 mutant plants are more tolerance to paraquat and salt due to the better protection from oxidative stress because of higher $A P X, G P X$, and $S O D$ activities (Figure 8). As an E3-ubiquitin ligase, PQT3 plays negative role in plants to achieve oxidative tolerance via selected degradation of histone modifying PROTEIN METHYLTRANSFERASE4b (PRMT4b) which is further involved in regulating GPX1 and APX1 expression (Luo et al., 2016). Therefore, the ospqt3 mutant plants performed better as indicated by their growth and yield under salt stress conditions compared with wild type plants (Figure 6). Secondly, the ospqt3 mutant plants had more tillers and larger panicle size compared to wild type plants (Figure 46). The grain yield is directly connected to panicle size and effective tiller number (Xing and Zhang, 2010). The mechanism by which the ospqt3 mutants improves the panicle size and effective tillers number is unclear yet but interesting to investigate in future.

\section{MATERIALS AND METHODS}

\section{Plant material and growth conditions}

Rice (Oryza sativa) wild type cv. Zhonghua11 along with three CRISPR/Cas edited Zhonghua11 mutants (ospqt3-1, ospqt3-2, and ospqt3-3) were used in this study (Hu et al., 
2016). Hydroponically grown plants were maintained in Kimura B solution (EHARA et al., 1990). Medium was refreshed on alternate days while growth conditions were maintained at $28^{\circ} \mathrm{C}$ temperature, photo-regime of 16 hours light -8 hours dark, $70 \%$ relative humidity, and light intensity at $200 \mu \mathrm{mol} \mathrm{m} \mathrm{s}^{-1}$.

\section{Seed germination}

Seeds of wild type, ospqt3 mutants were surface sterilized with $\left(0.1 \% \mathrm{HgCl}_{2}\right)$ for $15 \mathrm{~min}$ and then rinsed four times with sterile distilled water. To analyze seed germination, 50 70 seeds (three replicates per plant) were randomly placed in a bottle containing MS medium with paraquat or salt. All bottles were placed in a chamber that had an $8 \mathrm{~h}$ light $\left(200 \mu \mathrm{mol} \mathrm{m}{ }^{-2} \mathrm{~s}^{-}\right.$ $\left.{ }^{1}\right) / 16 \mathrm{~h}$ dark photoperiod at $28^{\circ} \mathrm{C}$. The seeds were considered to have germinated when their radicle or germ length reached approximately $1 \mathrm{~mm}$. Seed germination was observed daily to calculate the germination percentage (Wang et al., 2010).

\section{Salt treatment}

Wild type and mutant plants seeds, washed with distilled water, and were incubated at $37^{\circ} \mathrm{C}$ for 2 days. Germinated seeds were transferred to Hoagland solution ( $\mathrm{pH}$ 6) for 3 days followed by application of $75 \mathrm{mM}$ and $100 \mathrm{mM} \mathrm{NaCl}$ for 10 days. The provided growth conditions were kept at (14-h-light/10-h-dark cycle at $\left.28^{\circ} \mathrm{C}\right)$. For the salt treatment in soil, 30 seedlings from each of the wild type, ospqt3-1, ospqt3-2, and ospqt3-3 seedlings were directly sown on soil pot (the pot dimensions were $5 \times 5 \times 12 \mathrm{~cm}^{3}$, and five plants were grown per pot). After grown for 4 weeks in soil under greenhouse conditions of $16 \mathrm{~h} \mathrm{light/} 8 \mathrm{~h}$ dark) at $30^{\circ} \mathrm{C}$, plants were either irrigated with $0 \mathrm{mM}$ or $200 \mathrm{mM} \mathrm{NaCl}$ solution for 5-6 days.

For long term salt treatment in greenhouse, seeds of WT and ospqt3 mutant were germinated in plates for 4 days and then sown on similar pots which used previously for salt treatment of 4-week-old seedlings. Plants were watered with $70 \mathrm{mM} \mathrm{NaCl}$ every 4-5 days, we add only water when the salt precipitation increased in the tray that holds the pots (about twice a month). The plants were grown to mature under the conditions and yield data were collected.

\section{Gene expression analysis}

TRIZOL (Invitrogen) method was adopted for extraction of total cellular RNA while DNA was eliminated from extracted RNA through DNase I (TaKaRa). Using the cDNA synthesis kit (TaKaRa: Moloney Murine Leukemia Virus Version), cDNA was synthesized from 
extracted RNA. The synthesized cDNA was subjected into real-time $q P C R$ using OsACTIN1 as internal control. Primers used are enlisted in Table S1 of supplemental information.

\section{Enzyme activity assay}

For $O s A P X, O s G P X$, and $O s S O D$ enzyme activity assay, 16 days old rice seedlings treated hydroponically were crushed in liquid nitrogen, and re-suspended in pre-cooled enzyme extraction buffer. In vitro activities of $O s A P X, O s G P X$, and $O s S O D$ were measured according to the methods described by Nakano and Asada (Nakano and Asada, 1981), Halliwell and Foyer (Halliwell and Foyer, 1978), and Mizushima et al. (Mizushima et al., 1991), respectively. Subsequently, enzyme-coupled spectrophotometer assay kit (SKBC, China) was used as per manufacturer's guidelines to quantify the $O s A P X, O s G P X$, and $O s S O D$ activities.

\section{The field trials}

To test in field, plants were grown in paddy field in Hainan from December to April and in Hefei from June to August of each year. To evaluate yield components of ospqt3 (CRISPR) mutants with Zhonghua11 background (WT) in normal field environment, three T3homozygous independent lines of the ospqt 3 along with wild type were planted in paddy rice field, 3 plots per genotype, each plot contains 3 rows $\times 20$ plants per row along with wild type plants. These experiments were performed in two different locations in China: Hainan Island (December to April 2019) and Hefei (April to August 2019). To reduce the variability in field test, the plants in the edge were eliminated in each plot in order to avoid margin effects.

\section{Evaluation of agronomic traits}

Individual plant height, seed setting ratio, tiller number, number of seeds per panicle, and grain yield were measured according to protocol documented earlier (Hu et al., 2015).

\section{Supplemental Data}

Supplemental Table S1. Primers used in this study.

\section{Accession Numbers}

Sequence data from this article can be found in the database Rice Genome Annotation Project (https://rice.plantbiology.msu.edu/) under the following accession numbers: OsAPXI LOC_Os03g17690; OsPQT3 LOC_Os10g29560.1; OsAPx5, LOC_Os12g07830.1; OsAPx6, LOC_Os12g07820.1; OsAPx7,LOC_Os04g35520.2; OsGPX1,Os03g0358100; OsGPX3, 
LOC_Os11g18170.1; OsGPX5, LOC_Os02g44500.1; OsGPX7, LOC_Os06g08670.1;

OsACHT2, LOC_Os05g11090.1; OsCAT2, LOC_Os03g45170.1; OsCAT3,

LOC_OsO6g51150.1; OsCSD2/OsSOD2, LOC_OsO8g44770.1;

OsPRXQ,LOC_Os06g09610.1; OsGRX1,LOC_OsO2g30850; and Os2CPB,

LOC_Os04g33970.1.

\section{ACKNOWLEDGEMENTS}

This work was supported by the Strategic Priority Research Program of the Chinese Academy of Sciences (grant no. XDA24010303) and by grants from the National Natural

Science Foundation of China (grant no. 31770273), and Ministry of Science and Technology

of China (grant no. 2018ZX08009-11B, 2016ZX08005-004-003, 2016ZX08001003).

Alamin Alfatih and Sami Ullah Jan are recipients of CAS-TWAS President's Fellowship.

\section{CONFLICT OF INTEREST}

The authors declare no conflict of interest.

\section{AUTHOR CONTRIBUTIONS}

C.B.X. and A.A. designed the experiments. A.A. performed the experiments. S.U.J., Z.S.Z., and X.J.Q. participated in field trials and data analyses. A.A. wrote the manuscript. C.B.X. and W.J. revised the manuscript. C.B.X. supervised the project.

\section{REFERENCES}

Apel, K. and Hirt, H. (2004) Reactive oxygen species: metabolism, oxidative stress, and signal transduction. Annu. Rev. Plant Biol. 55, 373-399.

Boyer, J.S. (1982) Plant productivity and environment. Science 218, 443-448.

Chen, H., Ma, B., Zhou, Y., He, S.-J., Tang, S.-Y., Lu, X., Xie, Q., Chen, S.-Y. and Zhang, J.-S. (2018) E3 ubiquitin ligase SOR1 regulates ethylene response in rice root by modulating stability of Aux/IAA protein. Proceedings of the National Academy of Sciences 115, 4513-4518.

Chen, R.Q., Sun, S.L., Wang, C., Li, Y.S., Liang, Y., An, F.Y., Li, C., Dong, H.L., Yang, X.H., Zhang, J. and Zuo, J.R. (2009) The Arabid opsis PARAQUAT RESISTANT2 gene encodes an Snitrosoglutathione reductase that is a key regulator of cell death. Cell Research 19, 13771387.

Das, K. and Roychoudhury, A. (2014) Reactive oxygen species (ROS) and response of antioxidants as ROS-scavengers during environmental stress in plants. Frontiers in Environmental Science 2, 53.

Dixon, D.P. and Edwards, R. (2010) Glutathione transferases. The Arabidopsis Book/American Society of Plant Biologists 8. 
EHARA, H., TSUCHIYA, M. and OGO, T. (1990) Fundamental Growth Response to Fertilizer in Rice Plants: I. Varietal difference in the growth rate at the seedling stage. Japanese Journal of Crop Science 59, 426-434.

Gill, S.S. and Tuteja, N. (2010) Reactive oxygen species and antioxidant machinery in abiotic stress tolerance in crop plants. Plant physiology and biochemistry 48, 909-930.

Haley, T.J. (1979) Review of the toxicology of paraquat (1, 1'-dimethyl-4, 4'-bipyridinium chloride). Clinical toxicology 14, 1-46.

Halliwell, B. and Foyer, C. (1978) Properties and physiological function of a glutathione reductase purified from spinach leaves by affinity chromatography. Planta 139, 9-17.

Hu, B., Wang, W., Ou, S., Tang, J., Li, H., Che, R., Zhang, Z., Chai, X., Wang, H. and Wang, Y. (2015) Variation in NRT1. 1B contributes to nitrate-use divergence between rice subspecies. Nature Genetics 47, 834.

Hu, X., Wang, C., Fu, Y., Liu, Q., Jiao, X. and Wang, K. (2016) Expanding the range of CRISPR/Cas9 genome editing in rice. Molecular plant 9, 943-945.

Jamil, A., Riaz, S., Ashraf, M. and Foolad, M.R. (2011) Gene expression profiling of plants under salt stress. Critical Reviews in Plant Sciences 30, 435-458.

Kurepa, J., Smalle, J., Va, M., Montagu, N. and Inzé, D. (1998) Oxidative stress tolerance and longevity in Arabidopsis: the late-flowering mutant gigantea is tolerant to paraquat. The Plant Journal 14, 759-764.

Leshem, Y., Melamed-Book, N., Cagnac, O., Ronen, G., Nishri, Y., Solomon, M., Cohen, G. and Levine, A. (2006) Suppression of Arabidopsis vesicle-SNARE expression inhibited fusion of $\mathrm{H}_{2} \mathrm{O} 2-$ containing vesicles with tonoplast and increased salt tolerance. Proceedings of the National Academy of Sciences 103, 18008-18013.

Li, J.M., Su, Y.L., Gao, X.L., He, J., Liu, S.S. and Wang, X.W. (2011) Molecular characterization and oxidative stress response of an intracellular $\mathrm{Cu} / \mathrm{Zn}$ superoxide dismutase (CuZnSOD) of the whitefly, Bemisia tabaci. Archives of insect biochemistry and physiology 77, 118-133.

Li, X., Qian, Q., Fu, Z., Wang, Y., Xiong, G., Zeng, D., Wang, X., Liu, X., Teng, S. and Hiroshi, F. (2003) Control of tillering in rice. Nature 422, 618.

Lim, S.D., Cho, H.Y., Park, Y.C., Ham, D.J., Lee, J.K. and Jang, C.S. (2013) The rice RING finger E3 ligase, OsHCl1, drives nuclear export of multiple substrate proteins and its heterogeneous overexpression enhances acquired thermotolerance. Journal of experimental botany 64 , 2899-2914.

Luo, C., Cai, X.-T., Du, J., Zhao, T.-L., Wang, P.-F., Zhao, P.-X., Liu, R., Xie, Q., Cao, X.-F. and Xiang, C.-B. (2016) PARAQUAT TOLERANCE3 is an E3 ligase that switches off activated oxidative response by targeting histone-modifying PROTEIN METHYLTRANSFERASE4b. PLoS genetics 12, e1006332.

Marino, D., Froidure, S., Canonne, J., Khaled, S.B., Khafif, M., Pouzet, C., Jauneau, A., Roby, D. and Rivas, S. (2013) Arabidopsis ubiquitin ligase MIEL1 mediates degradation of the transcription factor MYB30 weakening plant defence. Nature communications 4, 1476.

Miller, G., Suzuki, N., Ciftci-Yilmaz, S. and Mittler, R. (2010) Reactive oxygen species homeostasis and signalling during drought and salinity stresses. Plant, cell \& environment 33, 453-467.

Mittler, R., Vanderauwera, S., Gollery, M. and Van Breusegem, F. (2004) Reactive oxygen gene network of plants. Trends in plant science 9, 490-498.

Mizushima, Y., Hoshi, K., Yanagawa, A. and Takano, K. (1991) Topical application of superoxide dismutase cream. Drugs under experimental and clinical research 17, 127-131.

Moon, J., Parry, G. and Estelle, M. (2004) The ubiquitin-proteasome pathway and plant development. The Plant Cell 16, 3181-3195.

Nakano, Y. and Asada, K. (1981) Hydrogen peroxide is scavenged by ascorbate-specific peroxidase in spinach chloroplasts. Plant and cell physiology 22, 867-880. 
Rockström, J. and Falkenmark, M. (2000) Semiarid crop production from a hydrological perspective: gap between potential and actual yields. Critical reviews in plant sciences 19, 319-346.

Summers, L.A. (1980) The bipyridinium herbicides:Academic Press Inc.

Takahashi, M. and Asada, K. (1988) Superoxide production in aprotic interior of chloroplast thylakoids. Archives of biochemistry and biophysics 267, 714-722.

Van Breusegem, F., Bailey-Serres, J. and Mittler, R. (2008) Unraveling the tapestry of networks involving reactive oxygen species in plants. Plant physiology 147, 978-984.

Vierstra, R.D. (2009) The ubiquitin-26S proteasome system at the nexus of plant biology. Nature Reviews Molecular Cell Biology 10, 385.

Wang, Z.-f., Wang, J.-f., Bao, Y.-m., Wang, F.-h. and Zhang, H.-s. (2010) Quantitative trait loci analysis for rice seed vigor during the germination stage. Journal of Zhejiang University Science B 11, 958-964.

$\mathrm{Xi}$, J., Xu, P. and Xiang, C.B. (2012) Loss of AtPDR11, a plasma membrane-localized ABC transporter, confers paraquat tolerance in Arabidopsis thaliana. Plant Journal 69, 782-791.

Xing, Y. and Zhang, Q. (2010) Genetic and Molecular Bases of Rice Yield. Annual Review of Plant Biology 61, 421-442.

Ye, B. and Gressel, J. (2000) Transient, oxidant-induced antioxidant transcript and enzyme levels correlate with greater oxidant-resistance in paraquat-resistant Conyza bonariensis. Planta 211, 50-61.

Yu, Y., Xu, W., Wang, J., Wang, L., Yao, W., Yang, Y., Xu, Y., Ma, F., Du, Y. and Wang, Y. (2013) The Chinese wild grapevine (Vitis pseudoreticulata) E3 ubiquitin ligase Erysiphe necator-induced RING finger protein 1 (EIRP1) activates plant defense responses by inducing proteolysis of the VpWRKY11 transcription factor. New Phytologist 200, 834-846.

Zhou, S., Chen, Q., Sun, Y. and Li, Y. (2017) Histone H2B monoubiquitination regulates salt stressinduced microtubule depolymerization in Arabidopsis. Plant, cell \& environment 40, 1512 1530. 


\section{Figure legends}

Figure 1. Identification of pqt3 mutant plants.

A. Schematic representation of OSPQT3 gene and location of CRISPR-Cas9 edited sites (indicated by triangles).

B. Sequence around CRISPR-Cas9 edited sites.

C. Sequencing confirmation of the edited site in the mutants.

\section{Figure 2. Loss-of-OsPQT3 confers paraquat and salt tolerance phenotypes in seed germination.}

A. Seed germination curve. Seeds of WT and pqt3 mutants were germianted on MS medium supplemented with 0 (Mock) or $0.1 \mu \mathrm{M}$ paraquat (PQ) and $175 \mathrm{mM} \mathrm{NaCl}$ for 8 days and germination \% was recorded everyday. Values are mean \pm SD (20 seeds per replicate, 4 replicates per treatment). Different letters denote significant differences $(\mathrm{P}<0.05)$ from Duncan's multiple range tests.

B. Seedling survival ratio. The above germinated seeds were grown for 2 weeks before seedling survival ratio was recorded. Values are mean \pm SD (20 plants per replicate, 4 replicates per treatment). Different letters denote significant differences $(\mathrm{P}<0.05)$ from Duncan's multiple range tests.

C. Growth of germinated seeds. Bar $=2 \mathrm{~cm}$.

Figure 3. Growth phenotype of ospqt3-1, ospqt3-2 and ospqt3-3 mutants in the presence of salt.

A. Seeds of WT and pqt3 mutants were germinated and tranferred to hydroponic culture for three days and then the medium was supplemented with $0 \mathrm{mM}$ (Mock), $75 \mathrm{mM}$ or $100 \mathrm{mM}$ $\mathrm{NaCl}$ and grown for 10 days before photographs were taken. $\mathrm{Bar}=2 \mathrm{~cm}$.

B. Survival ratio of the above genotypes and treatments. Values are mean \pm SD (24 plants per treatment, 3 replicates per treatment). Different letters denote significant differences ( $<$ 0.05) from Duncan's multiple range tests.

C. Salt tolerance assay in soil. The 4-week-old WT and ospqt3 mutants plants were watered by $0 \mathrm{mM}$ (Mock) or $200 \mathrm{mM} \mathrm{NaCl}$ for 5 days before photographs were taken. Bar $=10 \mathrm{~cm}$. 
D. Survival ratio of the above genotypes and treament. Values are mean \pm SD (5 plants per treatment, 6 replicates per treatment). Different letters denote significant differences $(\mathrm{P}<$ $0.05)$ from Duncan's multiple range tests.

Figure 4. The ospqt3 mutants exhibit improved yield in the field.

A. Growth status of WT (ZH11), ospqt3-1, ospqt3-2, and ospqt3-3 mutants plants in the field (Hefei, 2019). Bar $=10 \mathrm{~cm}$.

B. Representative panicles of the ospqt3 mutants and WT (ZH11). Bar $=4 \mathrm{~cm}$.

$\mathrm{C}-\mathrm{H}$. Yield components and plant height. Grain yield per single plant, tillers number per plant, panicles length, seeds setting rate, primary branch number per panicle, and plant height, respectively. Values are the mean \pm SD (30 plants per treatment, 3 replicates per treatment). Different letters denote significant differences $(\mathrm{P}<0.05)$ from Duncan's multiple range tests.

Figure 5. The repeat field trial confirmed the improved yield of ospqt3 mutants

A. Representative panicles of the ospqt3 mutant and WT (ZH11) plants grown in the filed (Hainan Island, 2019). Bar $=4 \mathrm{~cm}$.

B-G. Yield components and plant height. Grain yield per plant, tillers number per plant, panicles length, seeds setting rate, primary branch number per panicle, and plant height, respectively. Values are the mean \pm SD (30 plants per treatment, 3 replicates per treatment). Different letters denote significant differences $(\mathrm{P}<0.05)$ from Duncan's multiple range tests.

Figure 6. The ospqt 3 mutants exhibit enhanced yield under salt stress conditions.

A and B. Representative panicles of WT, ospqt3-1, ospqt3-2, and ospqt3-3 mutants plants grown in the greenhouse with or without $70 \mathrm{mM}$ salt as described in the Methods. Bar $=4 \mathrm{~cm}$. C-H. Yield components and plant height. Grain yield per plant, tillers number per plant, panicles numbers per plant, primary branch number per panicle, seeds setting rate and plant height, respectively. Values are the mean \pm SD ( 8 plants per treatment, 3 replicates per treatment). Different letters denote significant differences $(\mathrm{P}<0.05)$ from Duncan's multiple range tests.

Figure 7. The expression pattern and rapid down-regulation of $O S P Q T 3$ expression by various oxidative stress conditions. 
A. Expression pattern of $O S P Q T 3$ in different tissues of 3 development stages using quantitative RT-PCR. ACTIN is used as an internal reference gene. Values are the mean \pm SD ( $\mathrm{n}=3$ experiments).

B-G. Rapid down regulation of OsPQT3 by stress treatment. The transcript level of OsPQT3 was down-regulated by numerous oxidative stress: $5 \mu \mathrm{M}$ paraquat, $10 \mathrm{mM} \mathrm{H}_{2} \mathrm{O}_{2}$, mannitol, $200 \mathrm{mM} \mathrm{CdCl}_{2}$, drought, and $150 \mathrm{mM} \mathrm{NaCl}$. 1-week-old seedlings were used for these treatments and the RNA was extracted for quantitative RT-PCR analysis. Values are mean \pm $\mathrm{SD}(\mathrm{n}=3$ experiments). Different letters denote significant differences $(\mathrm{P}<0.05)$ from Duncan's multiple range tests.

\section{Figure 8. The expression of antioxidant enzymes is elevated in pqt3 mutants.}

A. Quantitative RT-PCR analysis of transcript levels of antioxidant enzyme genes. RNA samples were isolated from 7-day-old WT and ospqt3 seedlings for quantitative RT-PCR analysis. The transcript levels of OsAPX, OsGPX, OsSOD, OsCAT, OsMSD, OsGRXC, $O s P R X Q, O s 2 C P B$, and $O s A C H T$, were analyzed. Values are mean $\pm \mathrm{SD}$ ( $\mathrm{n}=3$ experiments). Different letters denote significant differences $(\mathrm{P}<0.05)$ from Duncan's multiple range tests. B. Enzyme activity of OsAPX, OsGPX, and OsSOD, in WT and ospqt3 mutants. Values are mean $\pm \mathrm{SD}(\mathrm{n}=3$ experiments $)$. Different letters denote significant differences $(\mathrm{P}<0.05)$ from Duncan's multiple range tests. 
A ospqt3-1 ospqt3-3

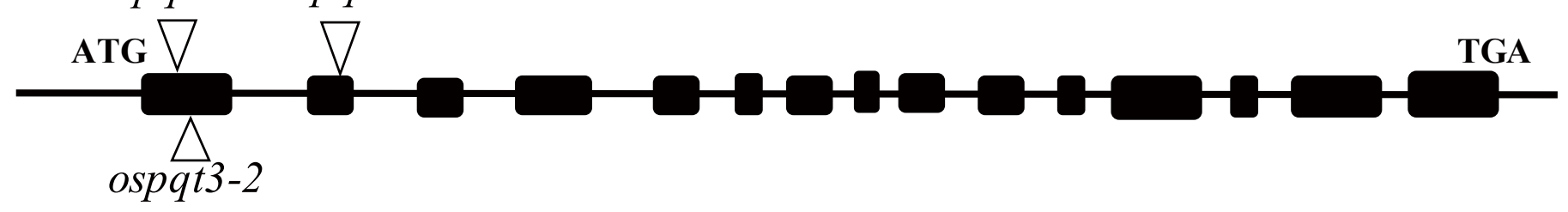

B

ospqt3-1 TGCCAGAGATTATGACTCGATTC-----GAGGGGCAATT

WT TGCCAgAgAtTATGACTCGATtCCGATCGAgGgGCAATT ospqt3-2 TGCCAGAGATTATGACTCGATTCCGATCAGAGGGGCAATT

WT TGCCAGAGATTATGACTCGATTCCGATC-GAGGGGCAATT ospqt3-3 ACACATCGGTGTTGATTCGTCGGATGACCAGGAAGACCAA WT ACACATCGGTGTTGATTCGTCGGAT-ACCAGGAAGACCAA sgRNA PAM

$\mathrm{C}$ PAM

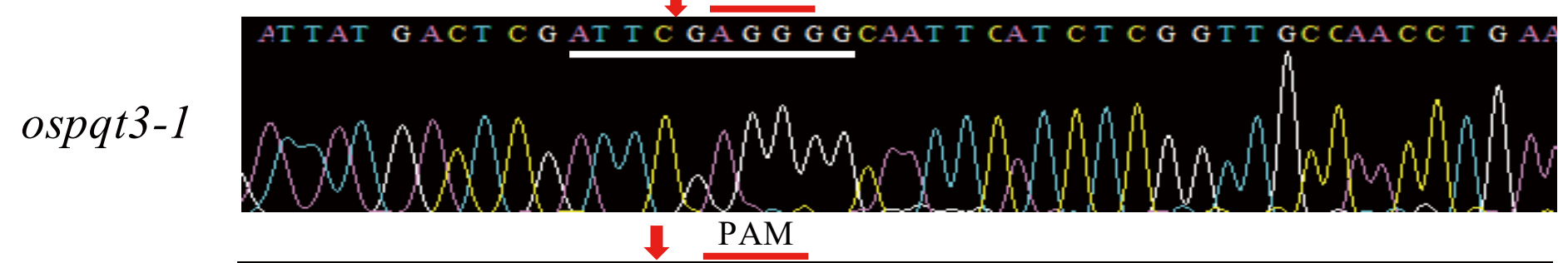

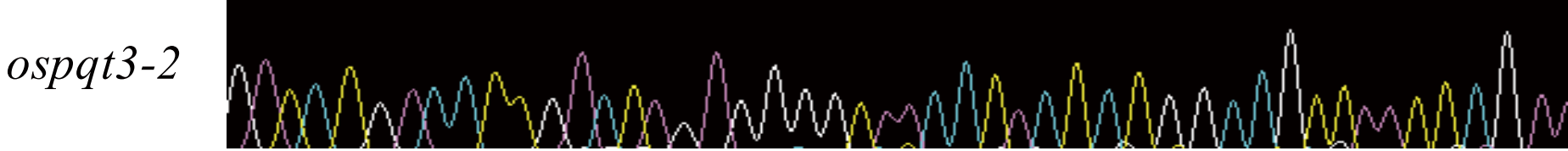
$1 \quad$ PAM

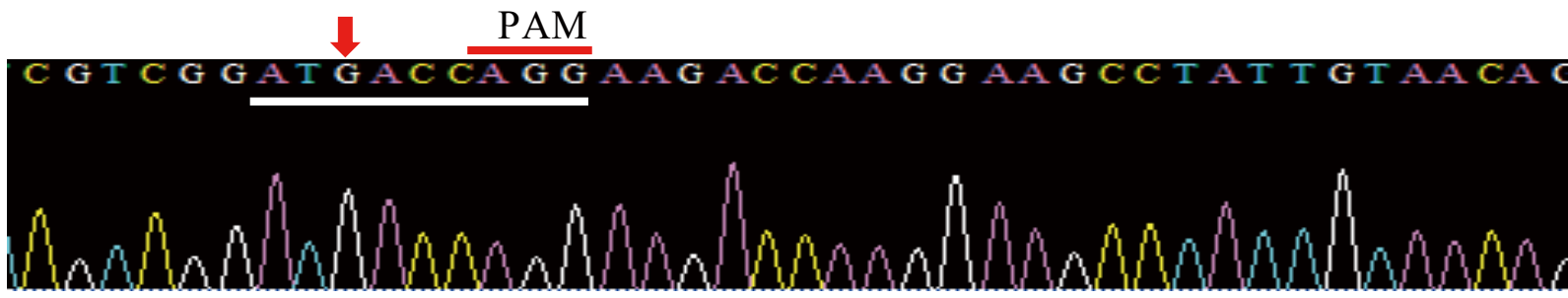

Figure 1. Identification of pqt3 mutant plants.

A. Schematic representation of OsPQT3 gene and location of CRISPR-Cas9 edited sites (indicated by triangles).

B. Sequence around CRISPR-Cas9 edited sites.

C. Sequencing confirmation of the edited site in the mutants. 
A
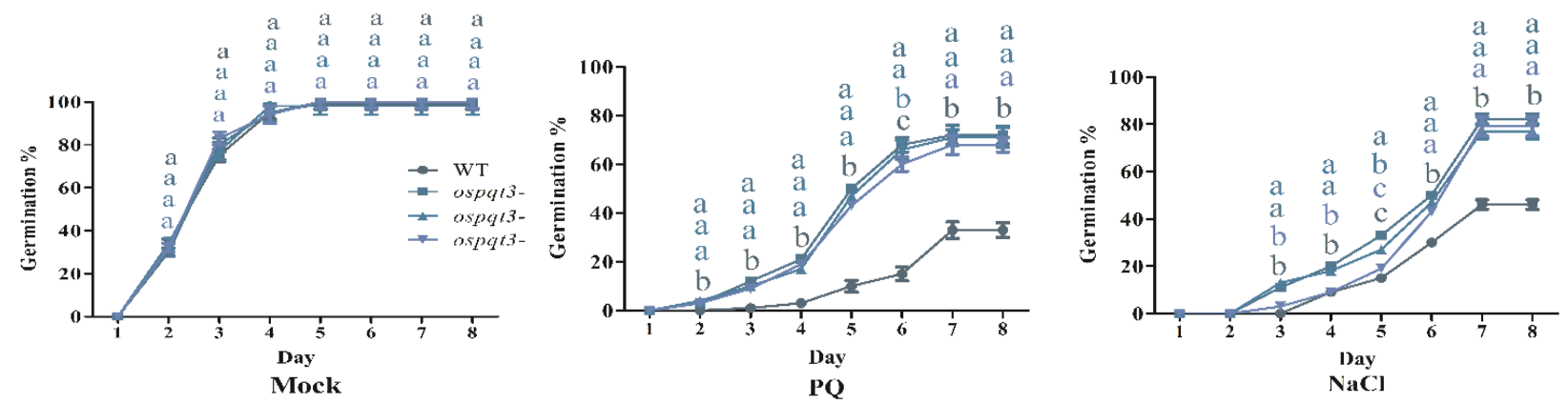

B
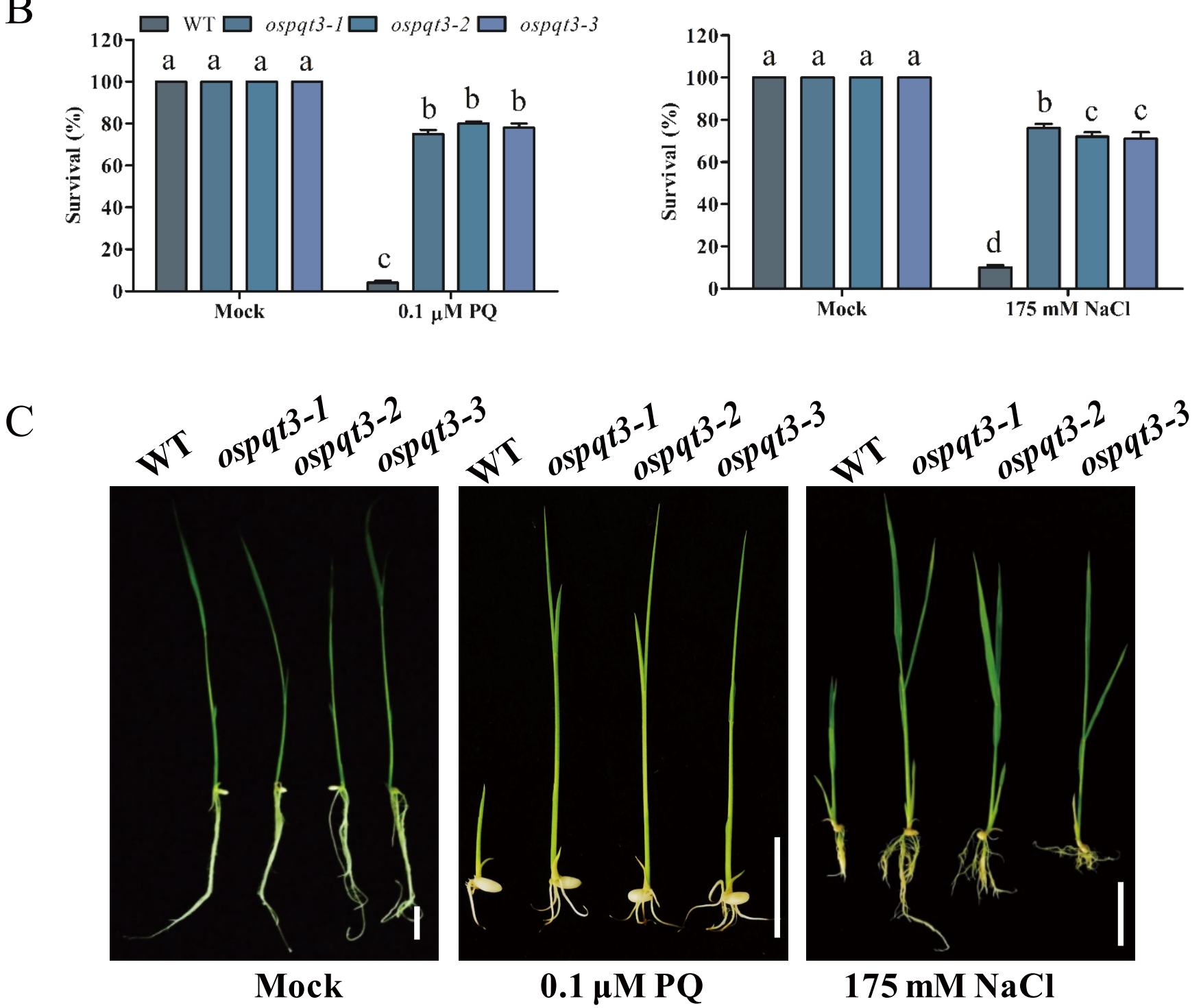

Figure 2. Loss-of-OsPQT3 confers paraquat and salt tolerance phenotypes in seed germination.

A. Seed germination curve. Seeds of WT and pqt3 mutants were germianted on MS medium supplemented with 0 or $0.1 \mu \mathrm{M}$ paraquat and $175 \mathrm{mM} \mathrm{NaCl}$ for 8 days and germination \% was recorded everyday. Values are mean \pm SD (20 seeds per replicate, 4 replicates per treatment). Different letters denote significant differences $(\mathrm{P}<0.05)$ from Duncan' s multiple range tests.

B. Seedling survival ratio. The above germinated seeds were grown for 2 weeks before seedling survival ratio was recorded. Values are mean \pm SD (20 plants per replicate, 4 replicates per treatment). Different letters denote significant differences $(\mathrm{P}<0.05)$ from Duncan' s multiple range tests.

C. Growth of germinated seeds. Bar $=2 \mathrm{~cm}$. 
A

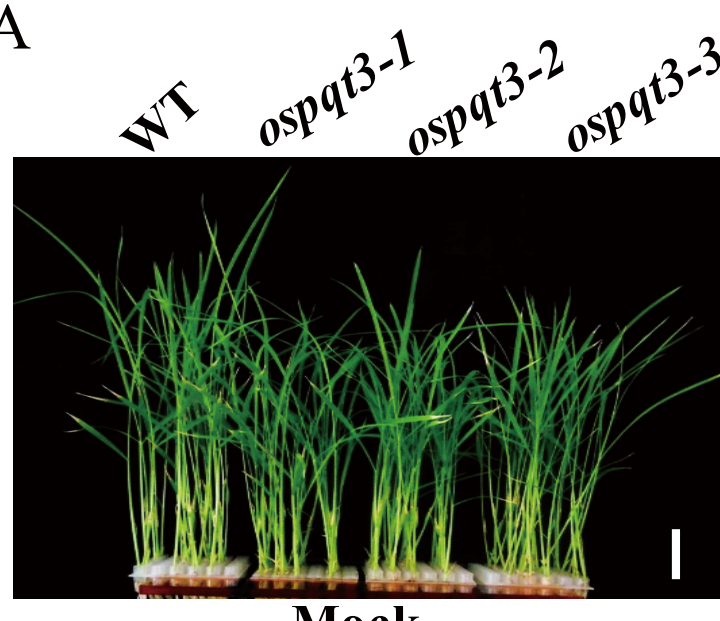

Mock

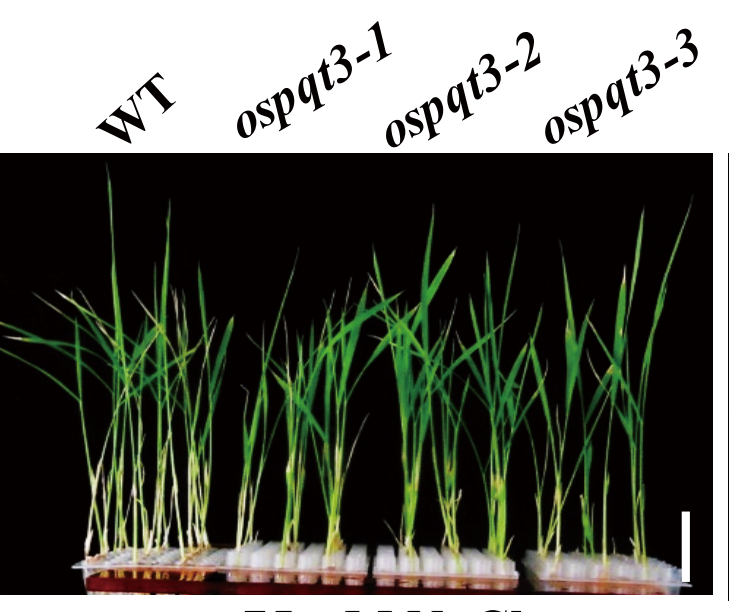

$75 \mathrm{mM} \mathrm{NaCl}$

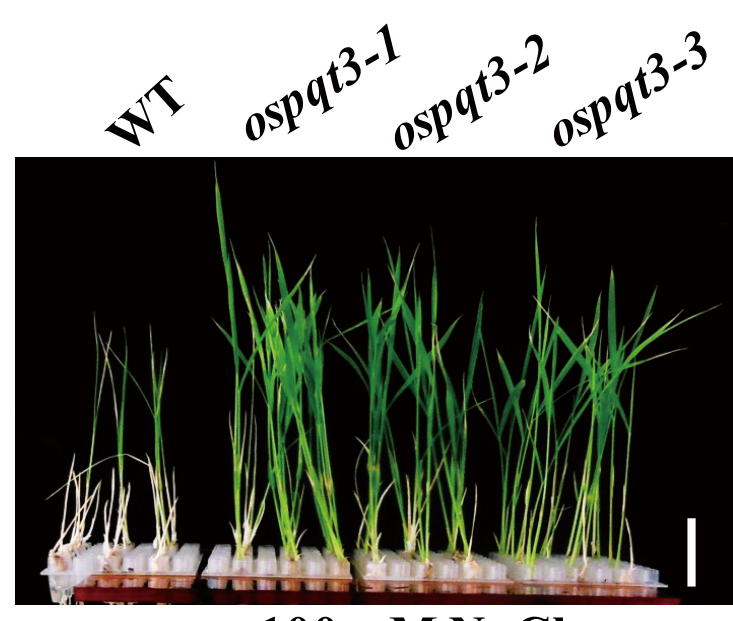

$100 \mathrm{mM} \mathrm{NaCl}$

B

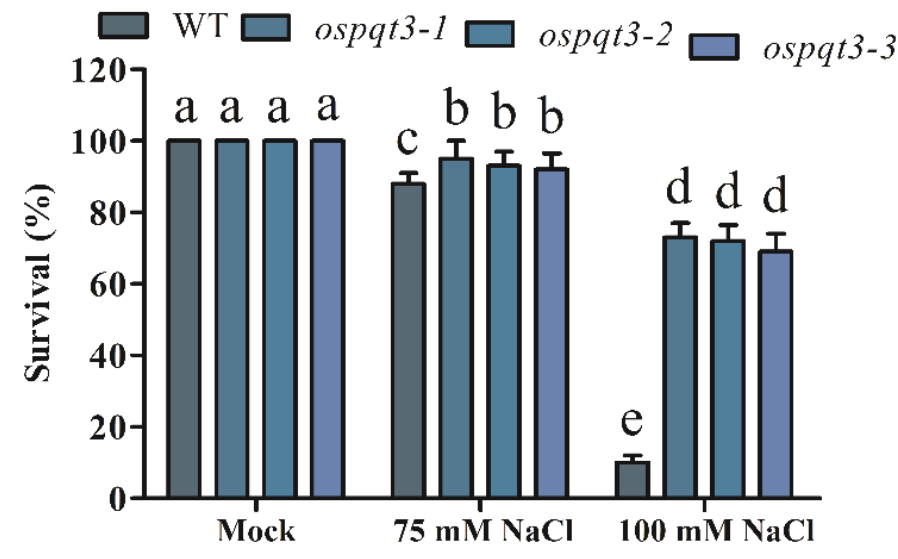

C

WT

ospqt3-1 ospqt3-2

ospqt3-3

WT

ospqt3-1

ospqt3-2

ospqt3-3

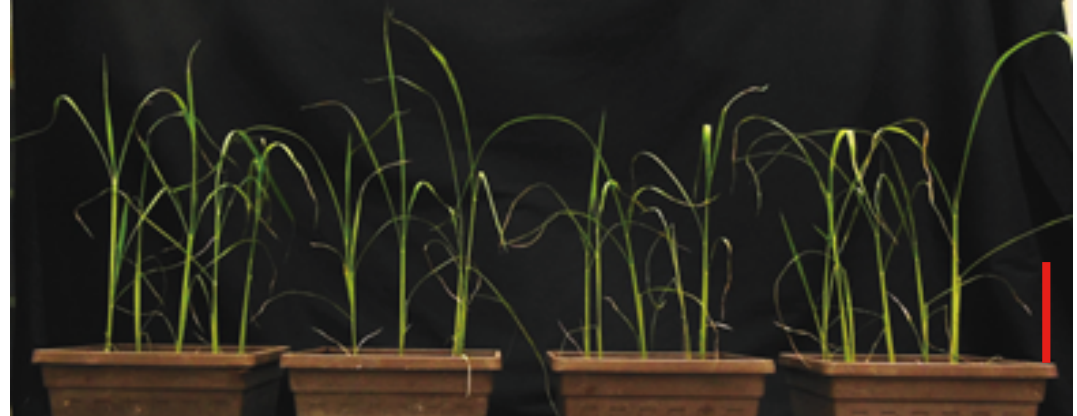

Mock

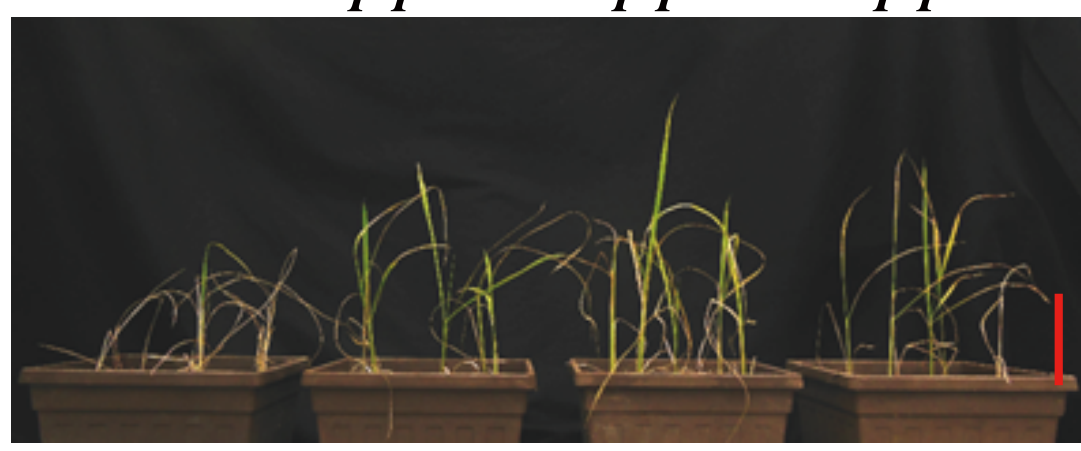

$200 \mathrm{mM} \mathrm{NaCl}$

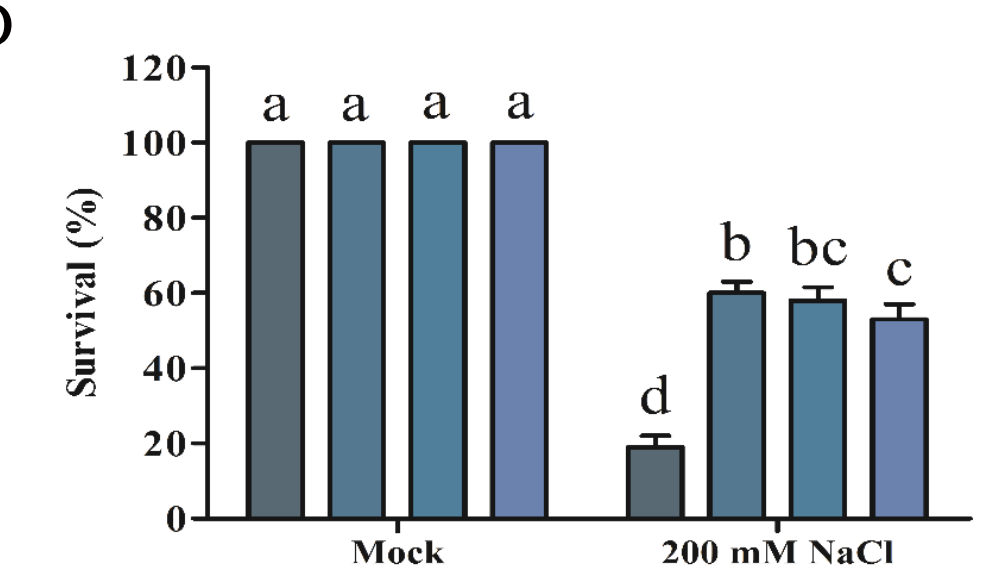

Figure 3. Growth phenotype of ospqt3-1, ospqt3-2 and ospqt3-3 mutants in the presence of salt.

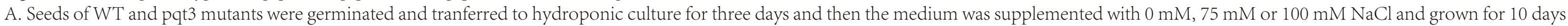
before photographs were taken. Bar $=2 \mathrm{~cm}$.

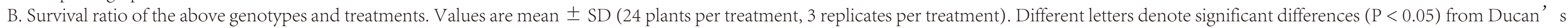
multiple range tests.

C. Salt tolerance assay in soil. The 4-week-old WT and ospqt 3 mutants plants were watered by $0 \mathrm{mM}$ or $200 \mathrm{mM} \mathrm{NaCl}$ for 5 days before photographs were taken. Bar $=10 \mathrm{~cm}$.

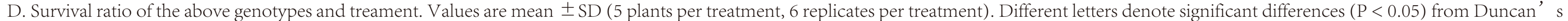
multiple range tests. 
A

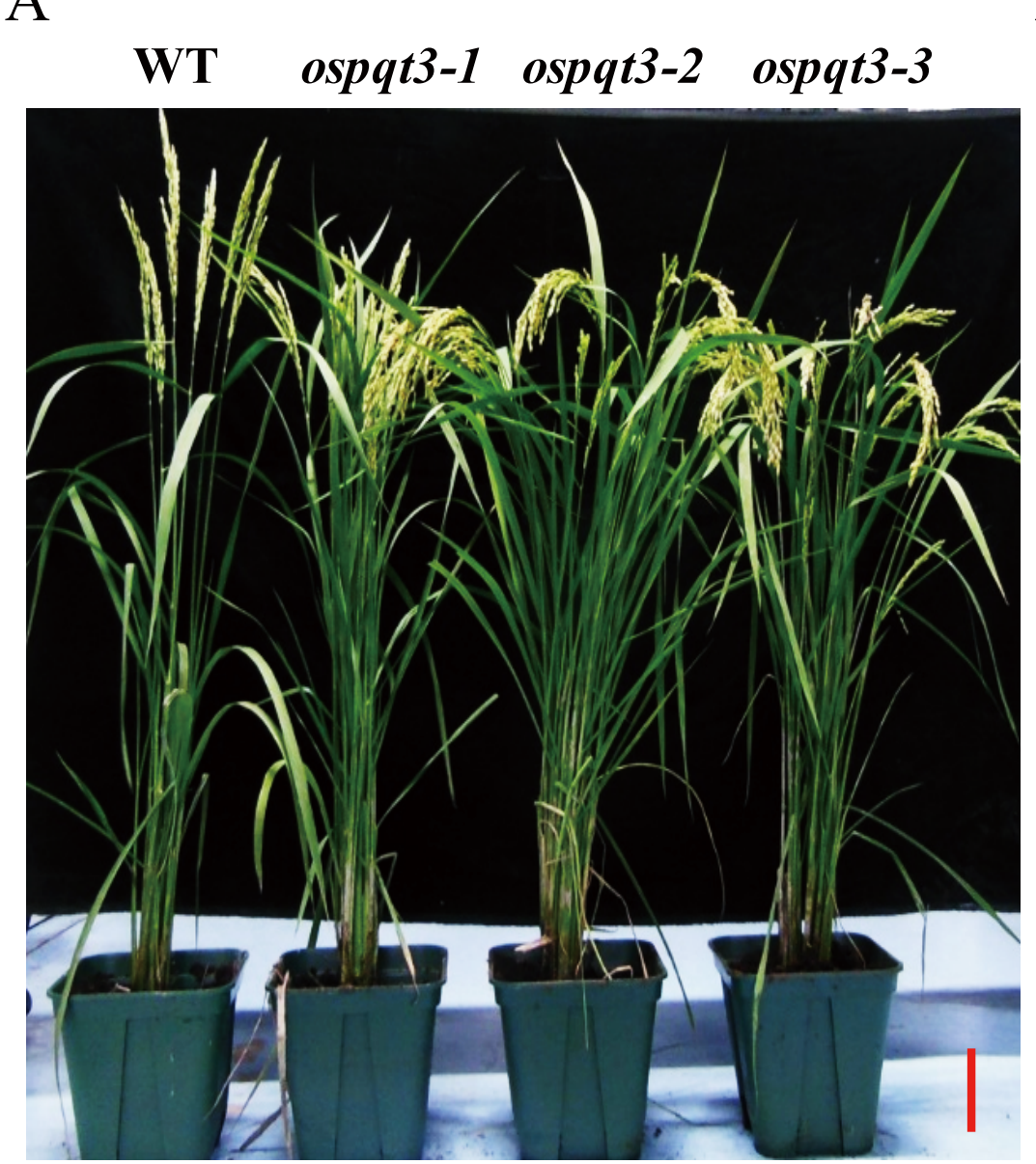

B

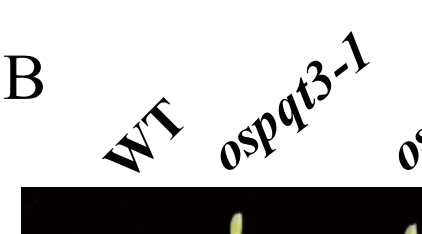

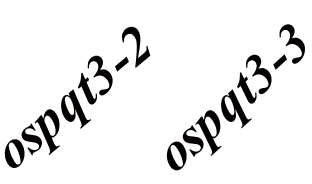

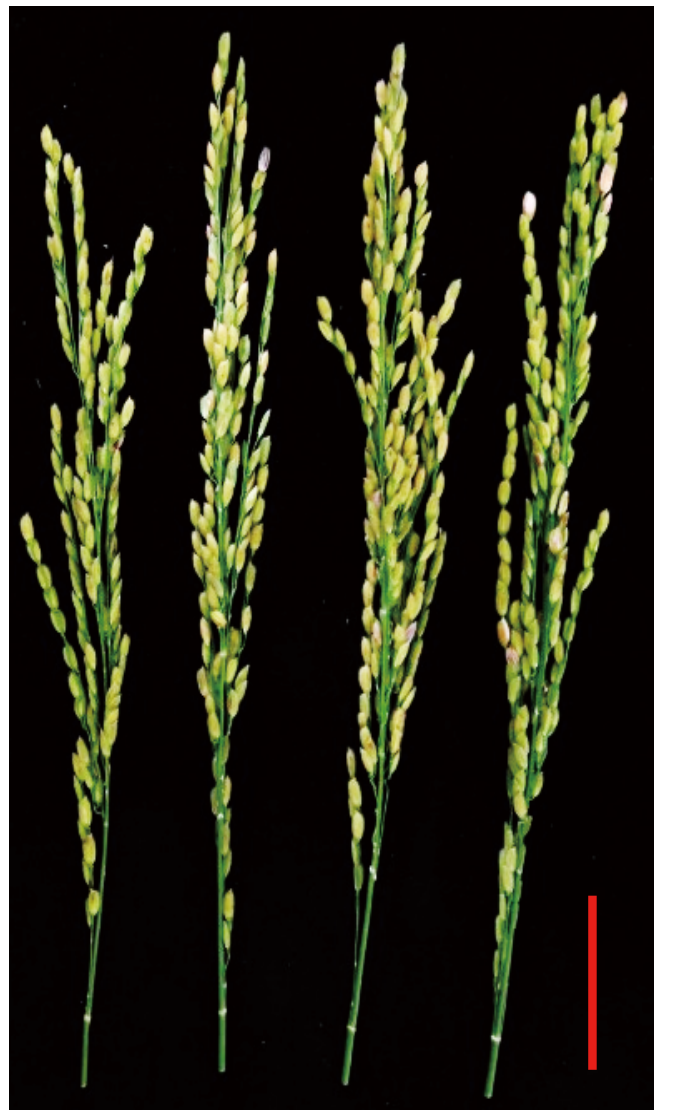

$\mathrm{C}$

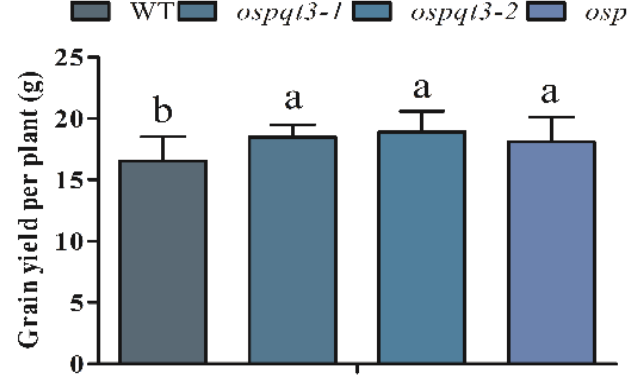

$\mathrm{D}$

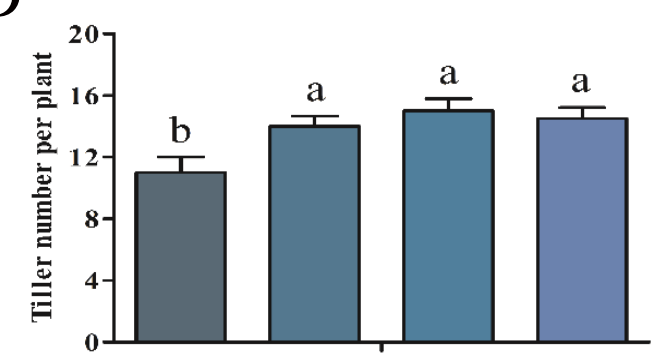

$\mathrm{E}$

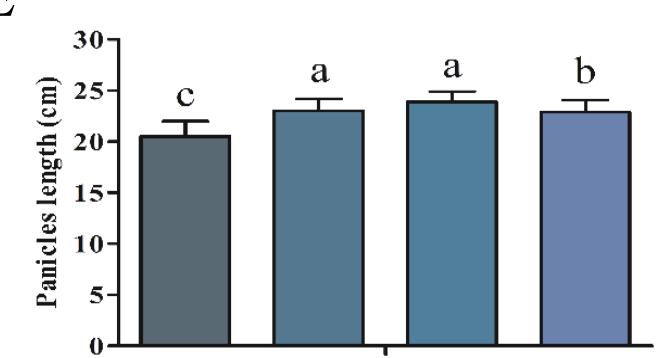

$\mathrm{F}$

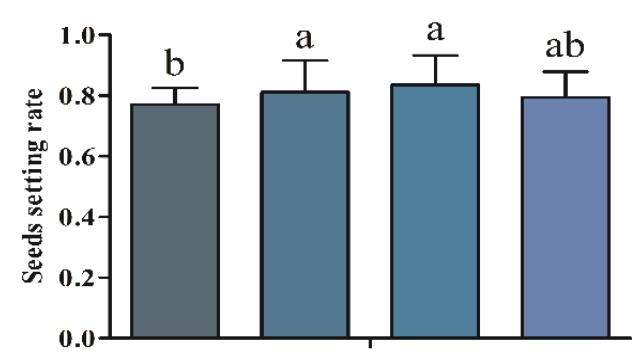

$\mathrm{G}$

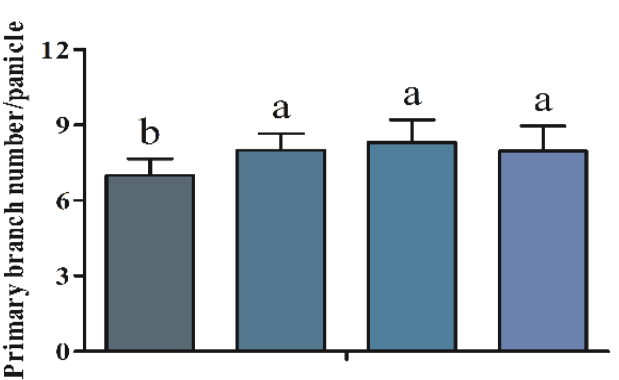

$\mathrm{H}$

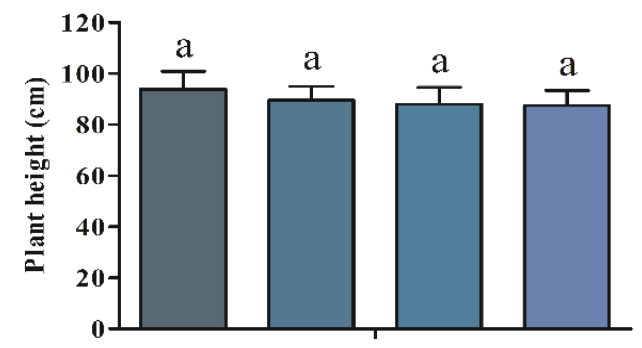

Figure 4. The ospqt3 mutants exhibit improved yield in the field.

A. Growth status of WT (ZH11), ospqt3-1, ospqt3-2, and ospqt3-3 mutants plants in the field (Hefei, 2019). Bar = $10 \mathrm{~cm}$.

B. Representative panicles of the ospqt3 mutants and WT ( $\mathrm{ZH11})$. Bar $=4 \mathrm{~cm}$.

C-H. Yield components and plant height. Grain yield per single plant, tillers number per plant, panicles length, seeds setting rate, primary branch number per panicle, and plant height, respectively. Values are the mean \pm SD (30 plants per treatment, 3 replicates per treatment). Different letters denote significant differences $(\mathrm{P}<0.05)$ from Duncan's multiple range tests. 
A

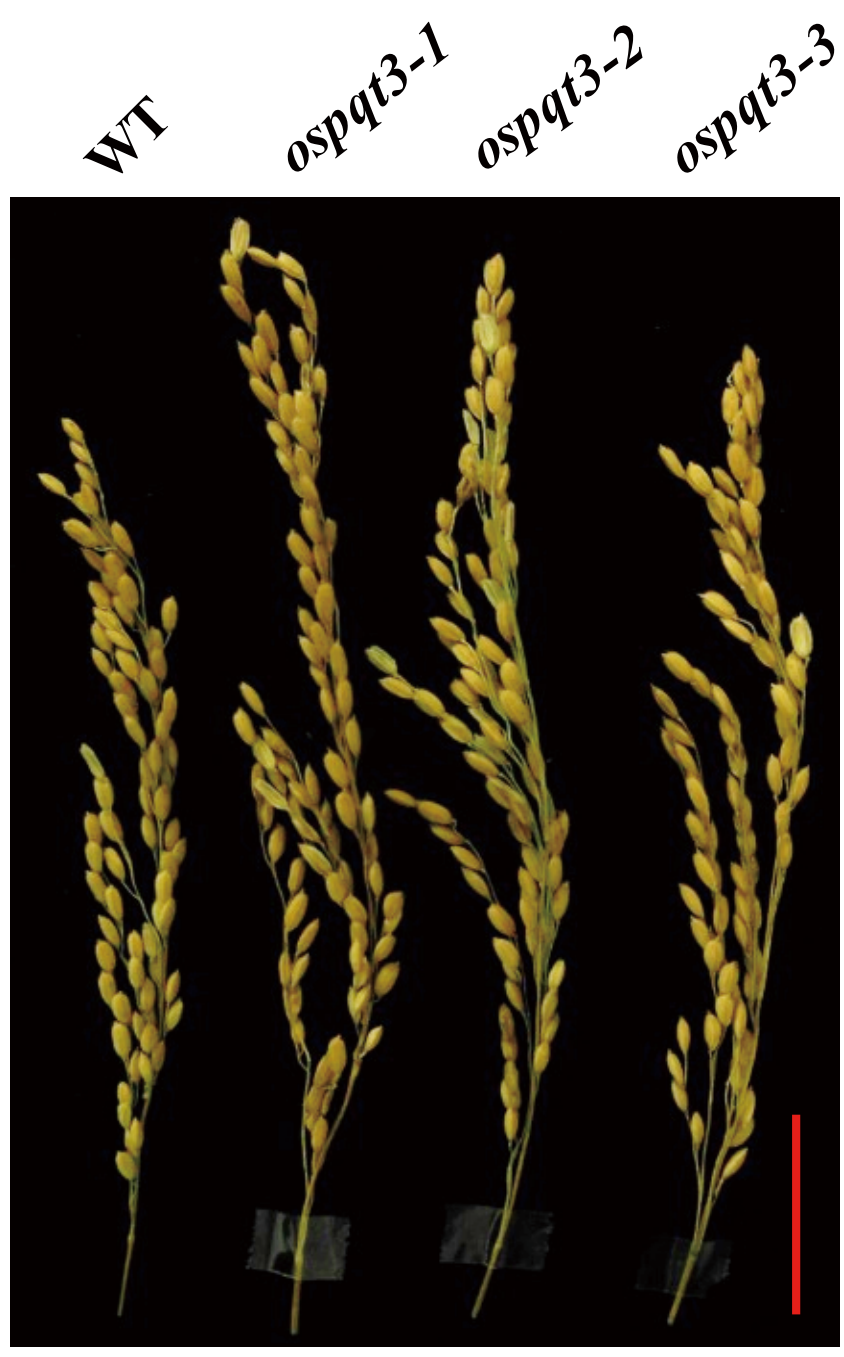

B

๒

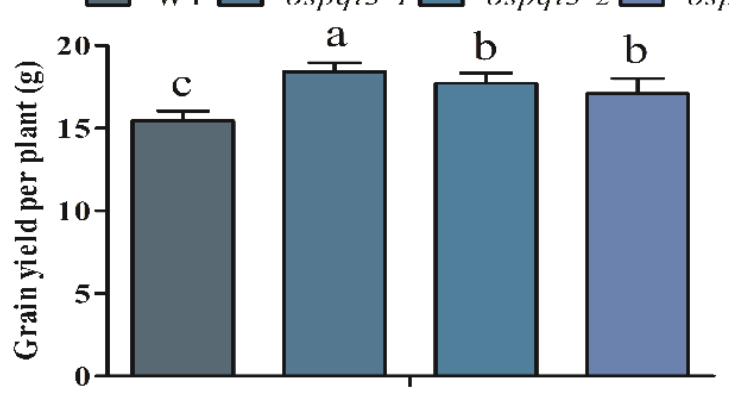

C

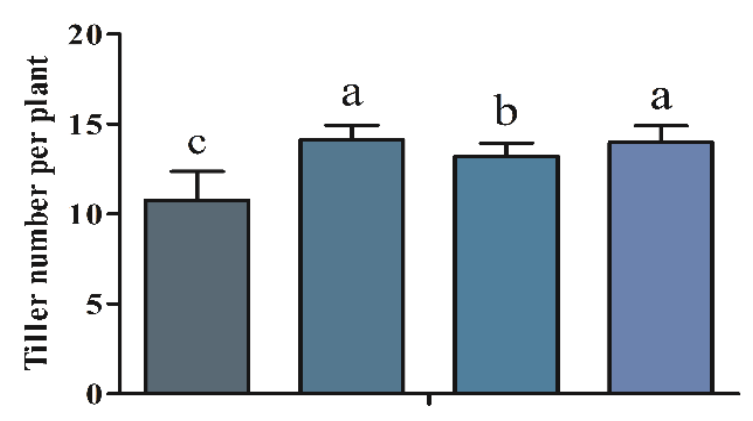

D

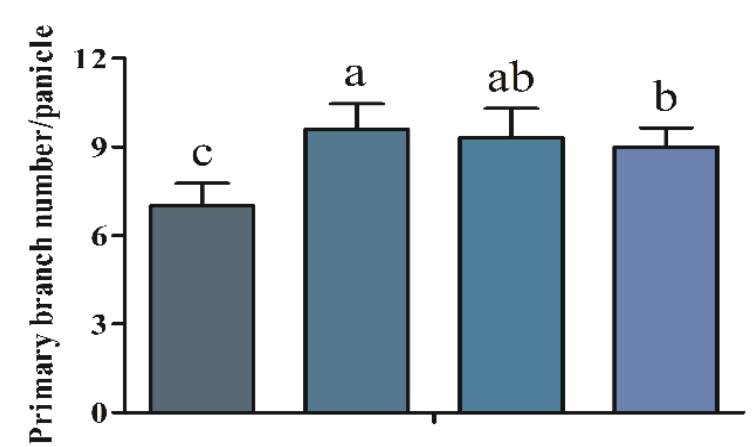

E

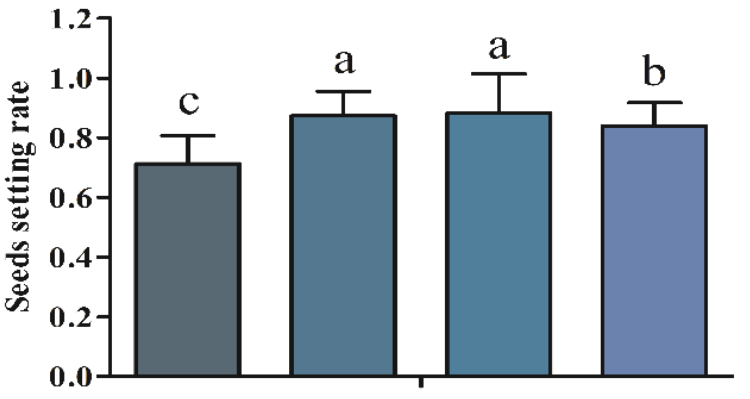

F

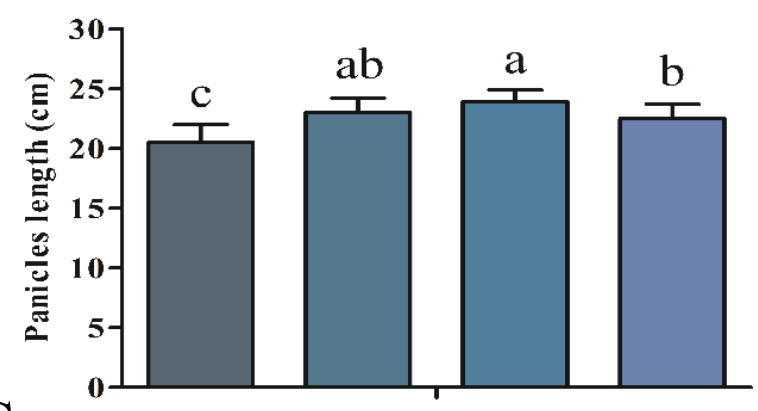

G

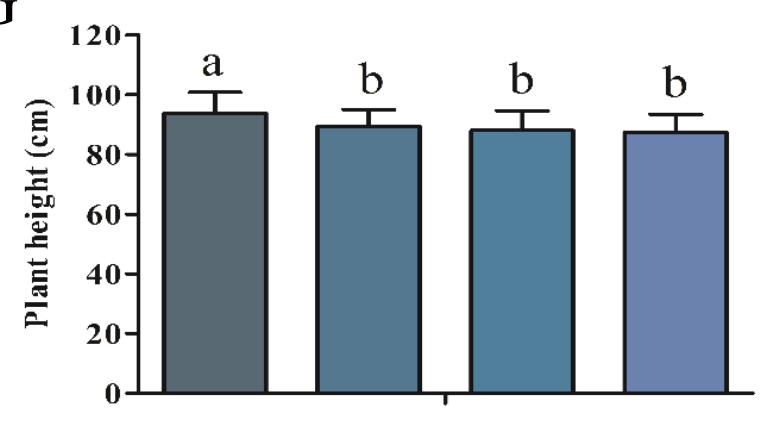

Figure 5. The repeat field trial confirmed the improved yield of ospqt3 mutants

A. Representative panicles of the ospqt3 mutant and WT (ZH11) plants grown in the filed (Hainan Island, 2019). Bar $=4 \mathrm{~cm}$.

B-G. Yield components and plant height. Grain yield per plant, tillers number per plant, panicles length, seeds setting rate, primary branch number per panicle, and plant height, respectively. Values are the mean \pm SD (30 plants per treatment, 3 replicates per treatment). Different letters denote significant differences (P $<0.05)$ from Duncan's multiple range tests. 
A

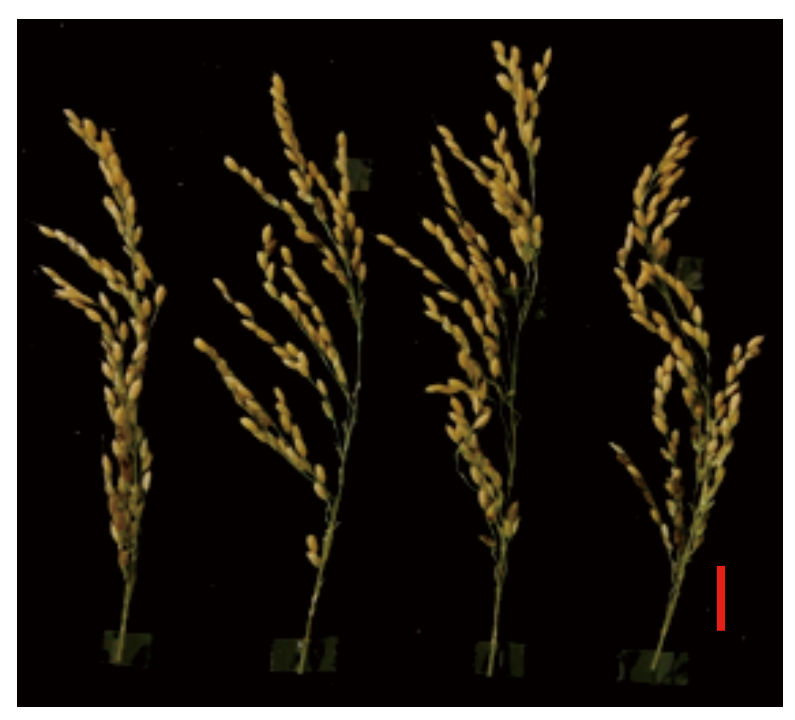

B WT ospqt3-1 ospqt3-2 ospqt3-3

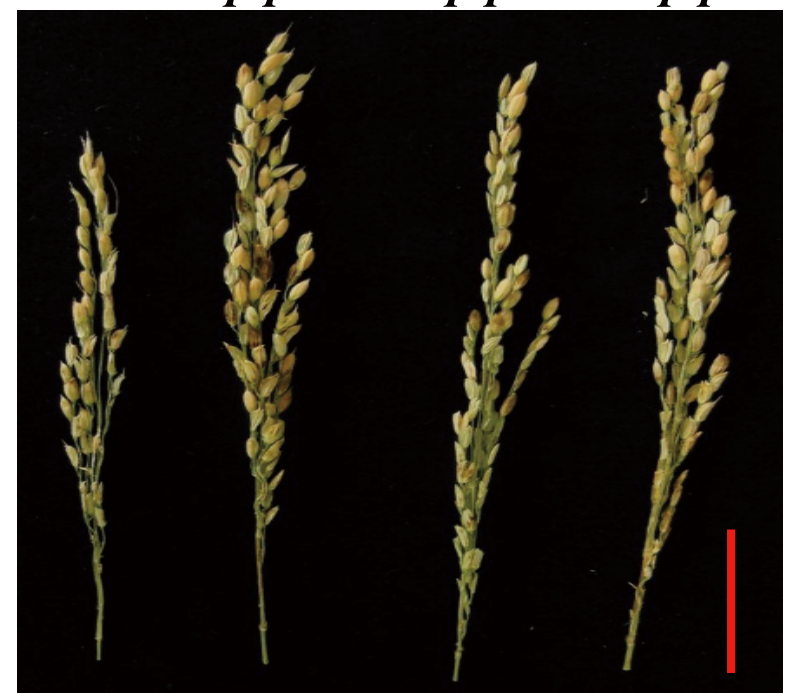

$70 \mathrm{mM} \mathrm{NaCl}$
C

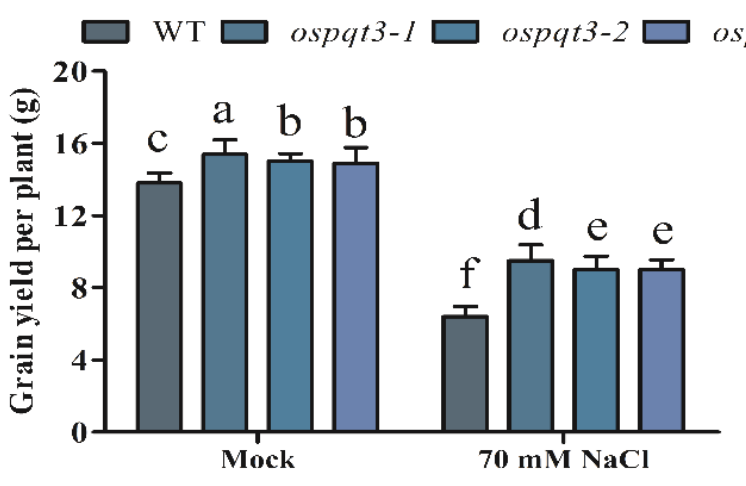

$\mathrm{D}$

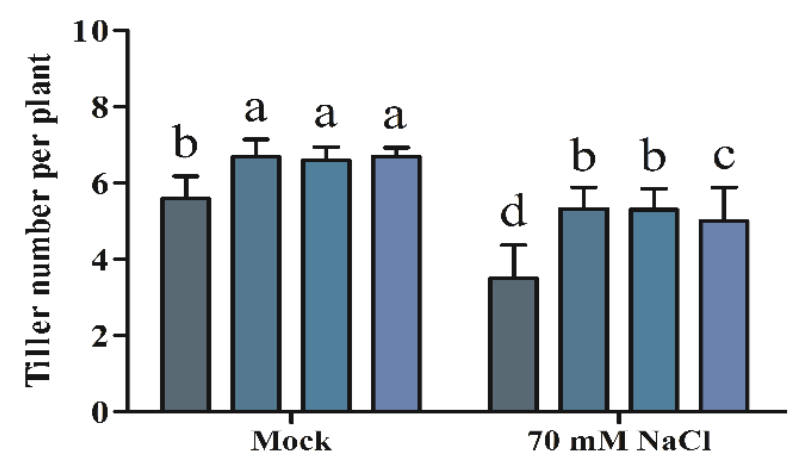

E

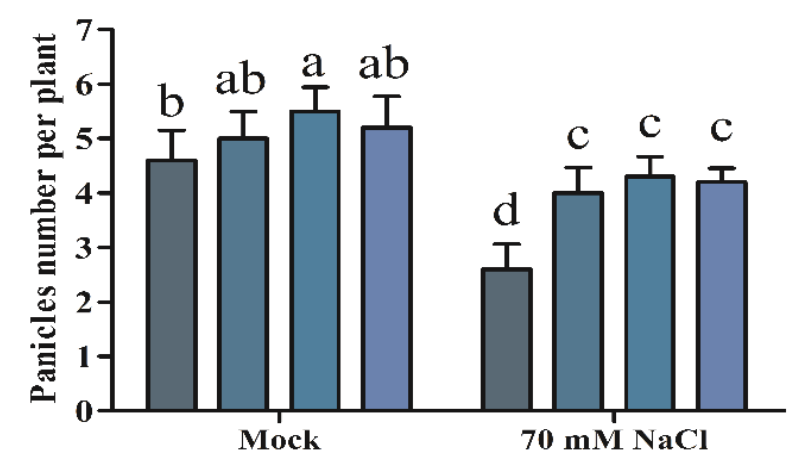

$\mathrm{F}$

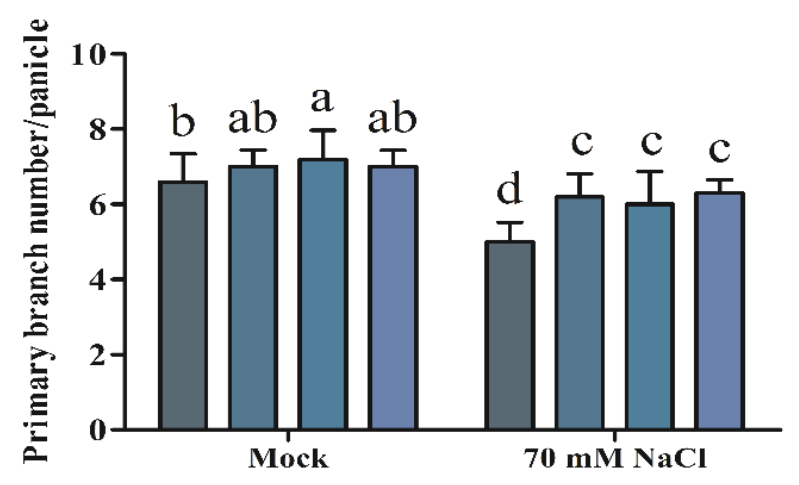

$\mathrm{G}$

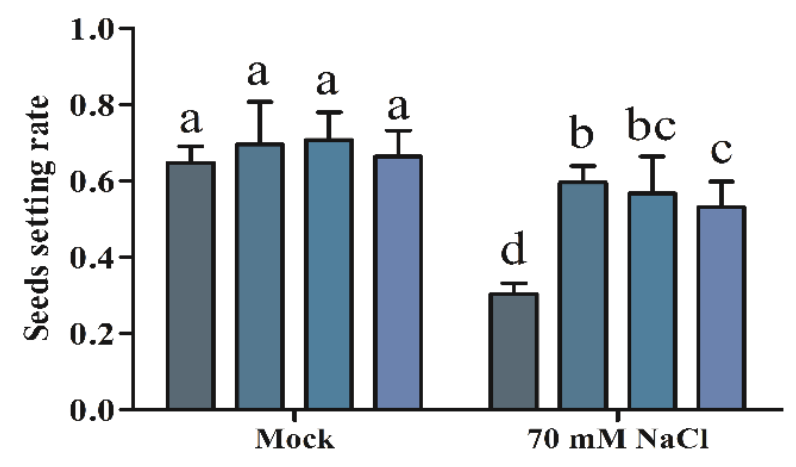

$\mathrm{H}$

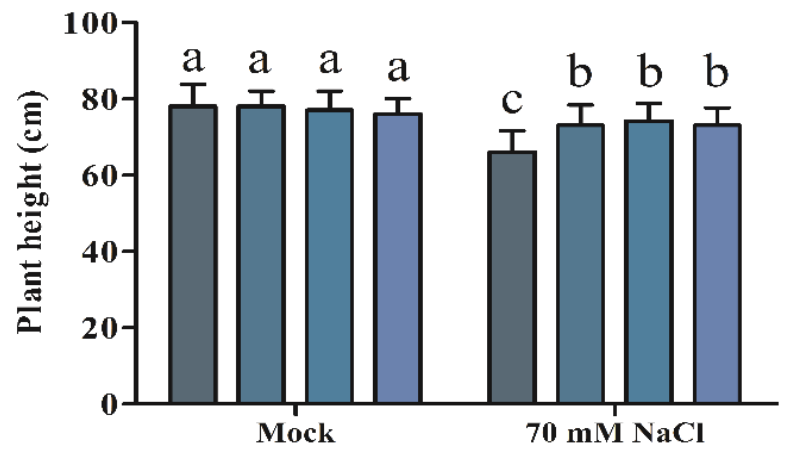

Figure 6. The ospqt3 mutants exhibit enhanced yield under salt stress conditions.

A and B. Representative panicles of WT, ospqt3-1, ospqt3-2, and ospqt3-3 mutants plants grown in the greenhouse with or without $70 \mathrm{mM}$ salt as described in the Methods. Bar $=4 \mathrm{~cm}$.

C-H. Yield components and plant height. Grain yield per plant, tillers number per plant, panicles numbers per plant, primary branch number per panicle, seeds setting rate and plant height, respectively. Values are the mean $\pm \mathrm{SD}$ (8 plants per treatment, 3 replicates per treatment). Different letters denote significant differences ( $\mathrm{P}<0.05)$ from Duncan's sultiple range tests. 
A

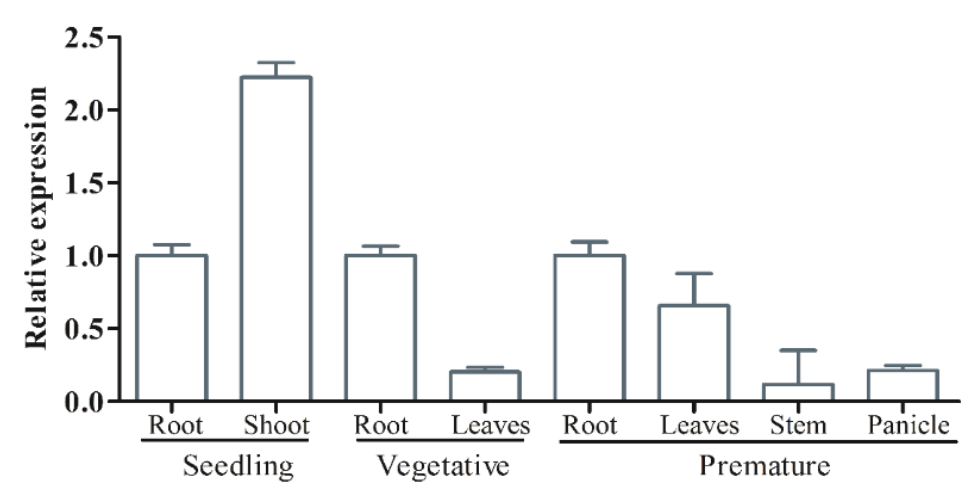

B

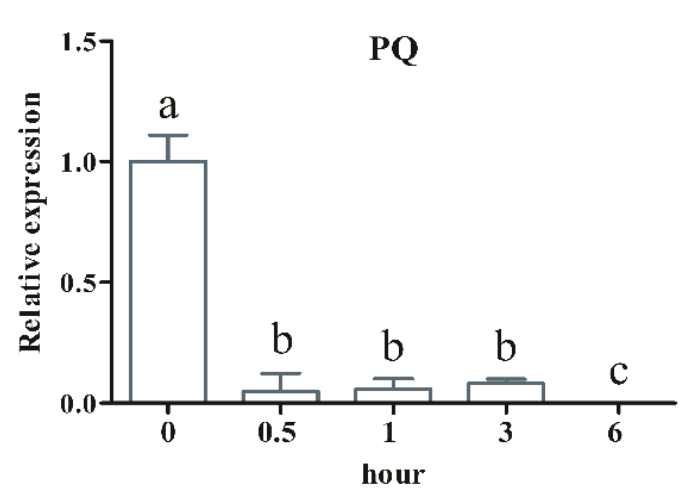

E

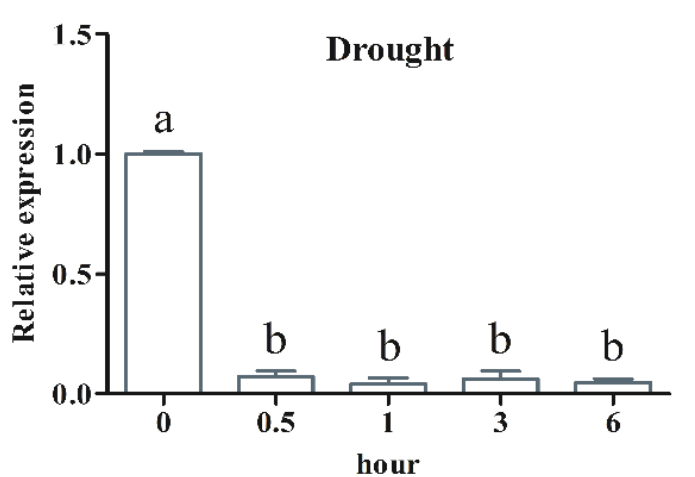

C

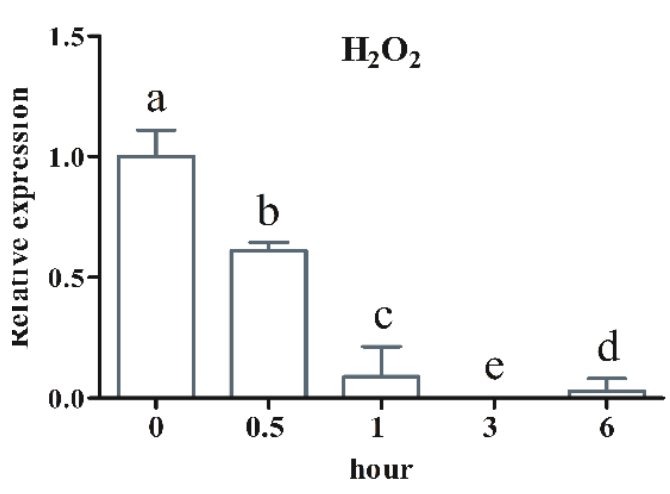

F

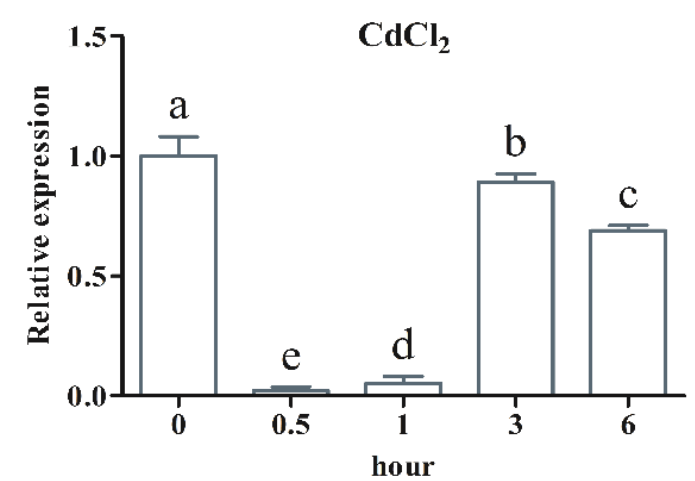

D

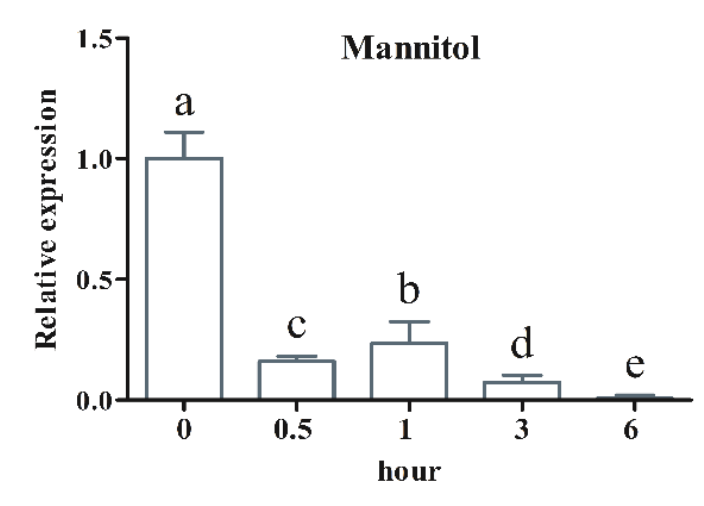

G

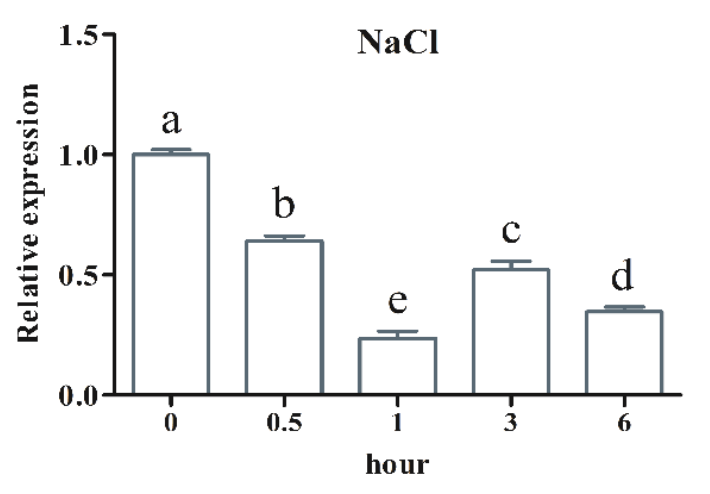

Figure 7. The expression pattern and rapid down-regulation of OsPQT3 expression by various oxidative stress conditions.

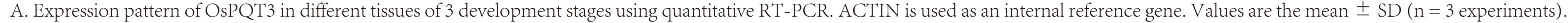

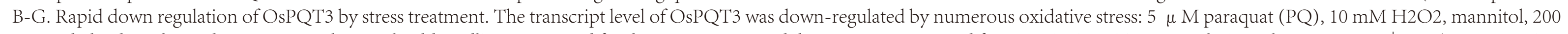

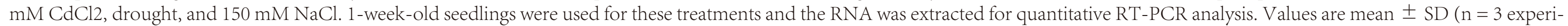
ments). Different letters denote significant differences $(\mathrm{P}<0.05)$ from Duncan’ s multiple range tests. 
A

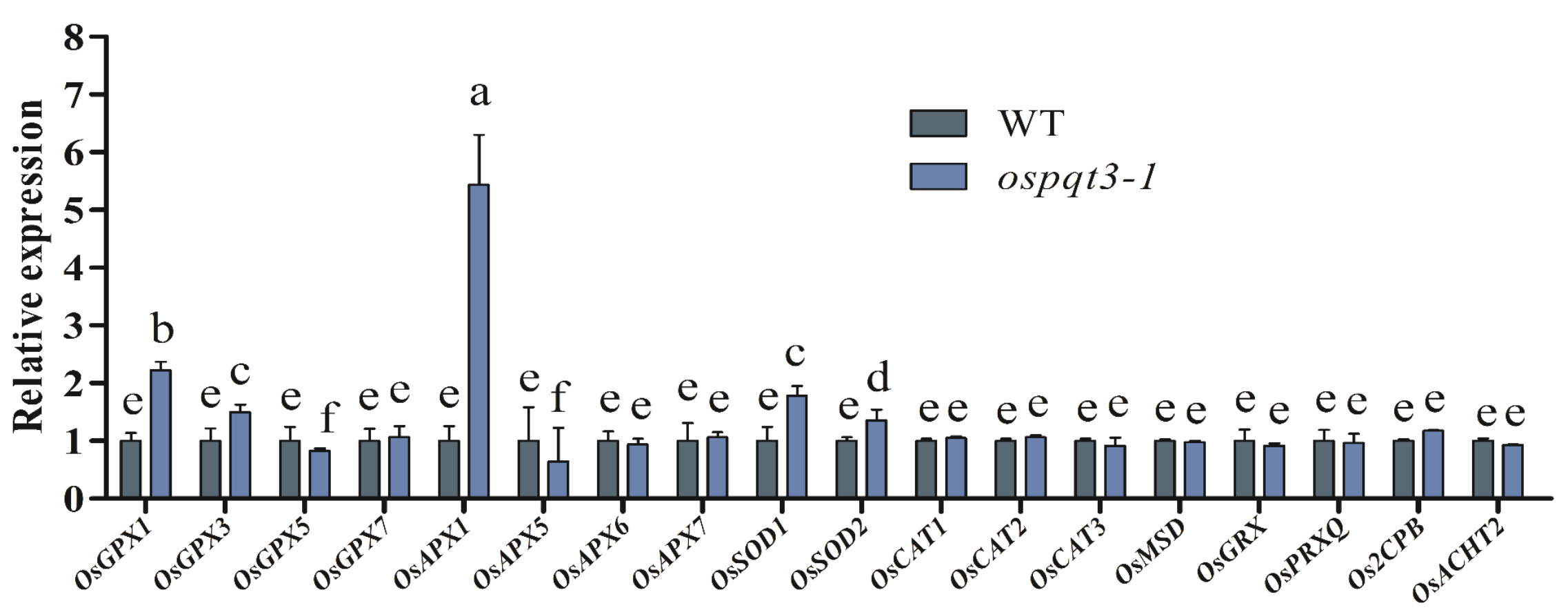

B
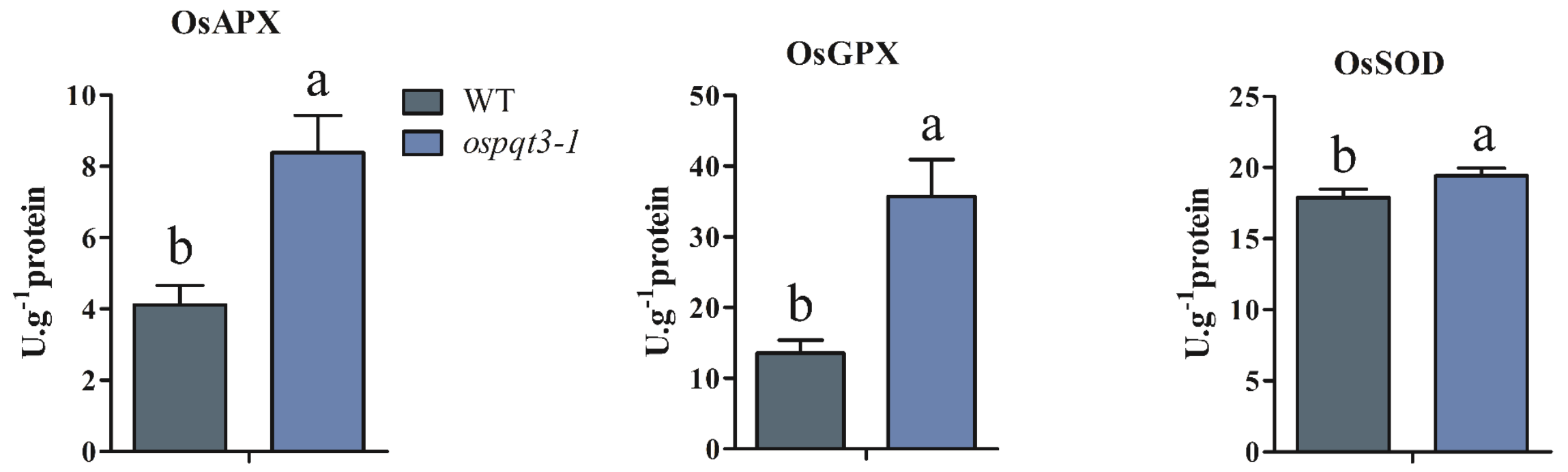

Figure 8. The expression of antioxidant enzymes is elevated in pqt3 mutants.

A. Quantitative RT-PCR analysis of transcript levels of antioxidant enzyme genes. RNA samples were isolated from 7-day-old WT and ospqt3 seedlings for quantitative RT-PCR analysis. The transcript levels of OsAPX, OsGPX, OsSOD, OsCAT, OsMSD, OsGRXC, OsPRXQ, Os2CPB, and OsACHT, were analyzed. Values are mean $\pm \mathrm{SD}(\mathrm{n}=3$ experiments $)$. Different letters denote significant differences $(\mathrm{P}<0.05)$ from Duncan' s multiple range tests.

B. Enzyme activity of OsAPX, OsGPX, and OsSOD, in WT and ospqt3 mutants. Values are mean \pm SD ( $\mathrm{n}=3$ experiments). Different letters denote significant differences $(\mathrm{P}<0.05)$ from Duncan’ s multiple range tests. 